\title{
Einstein Gravity, Lagrange-Finsler Geometry, and Nonsymmetric Metrics ${ }^{\star}$
}

\author{
Sergiu I. VACARU †‡ \\ $\dagger$ The Fields Institute for Research in Mathematical Science, \\ 222 College Street, 2d Floor, Toronto, M5T 3J1, Canada \\ $\ddagger$ Faculty of Mathematics, University "Al. I. Cuza” Iaşi, 700506, Iaşi, Romania \\ E-mail: Sergiu.Vacaru@gmail.com \\ URL: http://www.scribd.com/people/view/1455460-sergiu
}

Received June 24, 2008, in final form October 13, 2008; Published online October 23, 2008

Original article is available at http://www.emis.de/journals/SIGMA/2008/071/

\begin{abstract}
We formulate an approach to the geometry of Riemann-Cartan spaces provided with nonholonomic distributions defined by generic off-diagonal and nonsymmetric metrics inducing effective nonlinear and affine connections. Such geometries can be modelled by moving nonholonomic frames on (pseudo) Riemannian manifolds and describe various types of nonholonomic Einstein, Eisenhart-Moffat and Finsler-Lagrange spaces with connections compatible to a general nonsymmetric metric structure. Elaborating a metrization procedure for arbitrary distinguished connections, we define the class of distinguished linear connections which are compatible with the nonlinear connection and general nonsymmetric metric structures. The nonsymmetric gravity theory is formulated in terms of metric compatible connections. Finally, there are constructed such nonholonomic deformations of geometric structures when the Einstein and/or Lagrange-Finsler manifolds are transformed equivalently into spaces with generic local anisotropy induced by nonsymmetric metrics and generalized connections. We speculate on possible applications of such geometric methods in Einstein and generalized theories of gravity, analogous gravity and geometric mechanics.
\end{abstract}

Key words: nonsymmetric metrics; nonholonomic manifolds; nonlinear connections; Eisenhart-Lagrange spaces; generalized Riemann-Finsler geometry

2000 Mathematics Subject Classification: 53A99; 53B40; 53C21; 53C12; 53C44; 53Z05; 83C20; 83D05; 83C99

\section{Introduction}

The possibility that there are gravitational and matter field interactions described by nonsymmetric metrics has attracted attention beginning 1925, when A. Einstein [1, 2] proposed an unified theory of physical field (there were considered nonsymmetric metrics and complex tensor fields with Hermitian symmetry). Then, at the second step, L.P. Eisenhart investigated the properties of generalized Riemannin spaces enabled with nonsymmetric metrics [3, 4]. He put the problem to define the class of linear connections which are compatible with the symmetric part of a nonsymmetric metric (the Eisenhart problem). Further developments extended the problem to compatibility with general nonsymmetric metrics.

The third step of developments on nonsymmetric theory of gravity can be associated with J. Moffat works [5, 6] and further modifications proposed and outlined in [7, 8], generalization to noncommutative quantum gravity [9], and applications in modern cosmology [10, 11, 12].

The mentioned Eisenhart problem was solved in an important particular case in [13] and than approached in a form when the compatibility is with nonsymmetric metrics on generalized

\footnotetext{
*This paper is a contribution to the Special Issue "Élie Cartan and Differential Geometry". The full collection is available at http://www.emis.de/journals/SIGMA/Cartan.html
} 
Lagrange spaces (extending the Riemann-Finsler geometry) in [14], see a review of results, on our conventional forth direction in geometry and physics with nonsymmetric metrics, in Chapter 8 of monograph [15].

A set of additional arguments for geometric and physical models with nonsymmetric metrics follows from the theory of nonholonomic Ricci flows [16, 17] (the fifth direction of research with nonsymmetric metrics). It was mentioned that nonholonomic distributions on manifolds result in nonholonomic deformations of geometric objects (local frames, metrics, connections, functional and effective thermodynamical effects, ...) which define naturally new classes of canonical metric compatible linear connections with nonsymmetric Ricci tensor. Such nonsymmetric sources in the evolution equations for the nonholonomically constrained Ricci flows, in general, give rise to nonsymmetric components of metrics. Following certain geometric methods of generating exact solutions for Ricci flows of physically valuable metrics in gravity theories $[18,19,20,21,22]$, we constructed in explicit form [23] new classes of solutions defining evolutions of Taub NUT, solitonic and pp-waves and Schwarzschild metrics, in general relativity, into nonsymmetric metrics. It was concluded that nonholonomic spaces with nonsymmetric metrics and generalized connections arise naturally in modern geometry and physics if we try to describe a constrained dynamics and flow evolution of physical processes and geometrical/physical objects; a rigorous study of such theories presents a substantial interest.

The aim of this work is to elaborate a geometric approach to physical models and spaces enabled with nonholonomic (equivalently, nonintegrable/constrained) distributions and adapted frames with associated nonlinear connection structures and nonsymmetric metrics. We shall formulate a solution of the Eisenhart problem on nonholonomic manifolds ${ }^{1}$ when modelling of various types of generalizations, including those with nonsymmetric metrics and supersymmetric/spinor/noncommutative variables of the Riemann-Finsler and Lagrange-Hamilton geometries, is possible. This way, we shall provide a synthesis of the methods and ideas developed in directions two, three and four (mentioned above) in a general nonsymmetric metric compatible form, for various classes of linear and nonlinear connection, in strong relations to the fifth direction following the methods of geometry of nonholonomic manifolds. As general references on nonholonomic manifolds enabled with nonlinear connection structure, on the geometry of spaces with generic local anisotropy and applications to modern physics and mechanics, we cite the works $[24,25]$.

It should be emphasized that compatibility between the metric and general connection structures is very important not only for modelling physical theories in a more simplified geometrical form but also crucial for further both conceptual and technical developments of spinor analysis and theory of Dirac operators, on nonholonomic spaces with nonsymmetric metrics. A spinor formulation for nonsymmetric metrics and related nonholonomic manifolds will allow us to establish a connection with the geometry of nonholonomic Clifford bundles and algebroids [26, 27, 28], noncommutative and/or nonholonomic geometry and gravity [29, 30] and geometric quantization [31] developed following the methods of Lagrange and Finsler geometry and applied to the Einstein gravity and generalizations.

The work is organized as follows. In Section 2 we outline the algebraic properties of nonholonomic manifolds of even dimension enabled with nonlinear connection and nonsymmetric metric structures. The basic results from the differential geometry of distinguished connections compatible with nonsymmetric metrics are provided in Section 3. A metrization method for distinguished connections with nonsymmetric metrics is formulated in Section 4. Section 5 is devoted to the main theorems defining the set of distinguished connections being compatible with general nonsymmetric metric structures. In Section 6 we formulate the nonsymmetric gravity in terms of nonholonomic frames with associated nonlinear connection structure and distinguished linear connections; we emphasize the possibility to perform all constructions in metric

\footnotetext{
${ }^{1}$ For instance, on (pseudo) Riemannian manifolds enabled with nonholonomic distributions.
} 
compatible form. Some models of nonsymmetric Lagrange and Finsler spaces are analyzed in Section 7, where there are considered the conditions when such geometries can be generated by nonholonomic deformations of Einstein spaces and result in analogous modelling of gravitational effects by corresponding nonholonomic distributions. Conclusions and discussion are presented in Section 8. Some important local formulas are outlined in Appendix.

Remarks on notations and proofs of results. In this work, one follows the conventions from [24]. The Einstein's convention on summing "up" and "low" indices will be applied if the contrary will not be stated. We shall use "boldface" letters, $\mathbf{A}, \mathbf{B}^{\alpha}{ }_{\beta}, \ldots$ for geometric objects and spaces adapted to (provided with) a nonlinear connection structure. In general, small Greek indices are considered as abstract ones, which may split into horizontal (h) and vertical (v) indices, for instance $\alpha=(i, a), \beta=(j, b), \ldots$ where with respect to a coordinate basis they run values of type $i, j, \ldots=1,2, \ldots, n$ and $a, b, \ldots=n+1, n+2, \ldots, n+m$, for $n \geq 2$ and $m \geq 1$. There are introduced also left "up" and "low" labels of geometric objects: for instance,

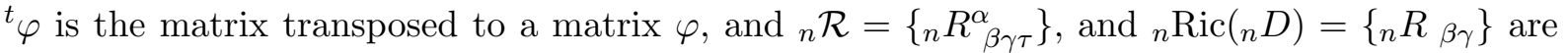
respectively the Riemannian and Ricci tensors for the normal d-connection ${ }_{n} \Gamma_{\beta \gamma}^{\alpha}$. We shall omit certain labels and indices if that will not result in ambiguities.

We shall not present detailed proofs if they can be obtained by local computations similar to those presented in $[24,25,15,7,8]$. The main difference is that in this article we work on nonholonomic manifolds with locally fibred structure defined by the nonlinear connection structure but not on tangent bundles or on usual Riemann-Cartan spaces and their nonsymmetric metric generalizations. With respect to certain classes of associated nonholonomic frames, the algebraic structure of formulas is similar to the case of integrable nonholonomic distributions. This allows us to simplify substantially the proofs of theorems even a formal dubbing into the so-called horizontal and vertical components exists.

\section{Nonsymmetric metrics on nonholonomic manifolds}

In this section we outline the algebraic properties of nonholonomic manifolds of even dimension enabled with nonlinear connection and nonsymmetric metric structures. We redefine on such spaces with local fibred structure the constructions from [13, 14, 15].

Let us consider a smooth manifold $\mathbf{V}^{n+n}$ of even dimension $n+n$ (in a particular case, for a tangent bundle, $\mathbf{V}^{n+n}=T M$, where $M$ is a smooth manifold of dimension $n$ ). We denote local coordinates in the form $u=(x, y)$, or $u^{\alpha}=\left(x^{i}, y^{a}\right)$, where indices $i, j, k, \ldots=1,2, \ldots, n$ and $a, b, c, \ldots=n+1, n+2, \ldots, n+n$ (on $T M$, we can use the same indices for both base and fiber indices).

Definition 2.1. A nonlinear connection (N-connection) structure $\mathbf{N}$ is defined by a nonholonomic (nonintegrable) distribution (a Whitney sum)

$$
T \mathbf{V}^{n+n}=h \mathbf{V}^{n+n} \oplus v \mathbf{V}^{n+n}
$$

into conventional horizontal (h) and vertical (v) subspaces.

In local form, a N-connection is given by its coefficients $N_{i}^{a}(u)$, for

$$
\mathbf{N}=N_{i}^{a}(u) d x^{i} \otimes \frac{\partial}{\partial y^{a}},
$$

stating on $\mathbf{V}^{n+n}$ a preferred frame (vielbein) structure

$$
\mathbf{e}_{\nu}=\left(\mathbf{e}_{i}=\frac{\partial}{\partial x^{i}}-N_{i}^{a}(u) \frac{\partial}{\partial y^{a}}, e_{a}=\frac{\partial}{\partial y^{a}}\right),
$$


and a dual frame (coframe) structure

$$
\mathbf{e}^{\mu}=\left(e^{i}=d x^{i}, \mathbf{e}^{a}=d y^{a}+N_{i}^{a}(u) d x^{i}\right) .
$$

The vielbeins (2.4) satisfy the nonholonomy relations

$$
\left[\mathbf{e}_{\alpha}, \mathbf{e}_{\beta}\right]=\mathbf{e}_{\alpha} \mathbf{e}_{\beta}-\mathbf{e}_{\beta} \mathbf{e}_{\alpha}=w_{\alpha \beta}^{\gamma} \mathbf{e}_{\gamma}
$$

with (antisymmetric) nontrivial anholonomy coefficients $w_{i a}^{b}=\partial_{a} N_{i}^{b}$ and $w_{j i}^{a}=\Omega_{i j}^{a}$, where

$$
\Omega_{i j}^{a}=\mathbf{e}_{j}\left(N_{i}^{a}\right)-\mathbf{e}_{i}\left(N_{j}^{a}\right)
$$

are the coefficients of N-connection curvature. The particular holonomic/integrable case is selected by the integrability conditions $w_{\alpha \beta}^{\gamma}=0 .^{2}$

Definition 2.2. A N-anholonomic manifold is a manifold enabled with N-connection structure (2.1).

The nonholonomic properties of a N-anholonomic manifold are completely defined by the the N-adapted bases (2.3) and (2.4). For instance, we can always transform an arbitrary manifold into a $\mathrm{N}$-anholonomic one by enabling it with a set of coefficients $N_{i}^{a}$ for a corresponding N-connection structure. Equivalently, we can say that such coefficients define a class of (linearly depending on $N_{i}^{a}$ ) linear frames. A geometric/physical motivation for such constructions can be provided if, for instance, the N-coefficients are induced by a (regular) Lagrange structure (in geometric mechanics, see $[25,15]$ ), or by certain off-diagonal coefficients of symmetric and/or nonsymmetric metrics, see examples and details in [24, 30, 17, 23]. A manifold is not $\mathrm{N}$-anholonomic if it is enabled with a trivial $\mathrm{N}$-connection structure when the corresponding nonholonomic coefficients vanish.

One says that a geometric object is N-adapted (equivalently, distinguished), i.e. a d-object, if it can be defined by components adapted to the splitting (2.1) (one uses terms d-vector, dform, d-tensor $)^{3}$. For instance, a d-vector is written in the form $\mathbf{X}=X^{\alpha} \mathbf{e}_{\alpha}=X^{i} \mathbf{e}_{i}+X^{a} e_{a}$ and a one d-form $\widetilde{\mathbf{X}}$ (dual to $\mathbf{X}$ ) is $\widetilde{\mathbf{X}}=X_{\alpha} \mathbf{e}^{\alpha}=X_{i} e^{i}+X_{a} e^{a}$. On N-anholonomic manifold it is convenient to work with d-objects because in this case all geometric and physical objects are derived in a form adapted to the $\mathrm{N}$-connection structure (i.e. to the corresponding class of imposed nonholonomic constraints). Of course, any d-tensor can be transformed into a general tensor but in such a case we "do not take care" about existing N-connection structure.

There is an almost complex structure $\mathbb{F}$ associated to a prescribed $\mathrm{N}$-connection structure $\mathbf{N}$, which is defined by operators

$$
\begin{aligned}
& \mathbb{F}\left(\mathbf{e}_{i}=\frac{\partial}{\partial x^{i}}-N_{i}^{a} \frac{\partial}{\partial y^{a}}\right)=-e_{i}=-\frac{\partial}{\partial y^{i}}, \\
& \mathbb{F}\left(e_{i}=\frac{\partial}{\partial y^{i}}\right)=\mathbf{e}_{i}=\frac{\partial}{\partial x^{i}}-N_{i}^{a} \frac{\partial}{\partial y^{a}}
\end{aligned}
$$

and has the property $\mathbb{F}^{2}=-I$, where $I$ is the identity matrix.

In this work, we study the geometric properties of spaces $\left(\check{g}_{i j}, \mathbf{V}^{n+n}, \mathbf{N}\right)$, where the h-subspace is enabled with a nonsymmetric tensor field (metric) $\check{g}_{i j}=g_{i j}+a_{i j}$, where the symmetric part $g_{i j}=g_{j i}$ is nondegenerated and $a_{i j}=-a_{j i}$.

\footnotetext{
${ }^{2}$ we use boldface symbols for spaces (and geometric objects on such spaces) enabled with N-connection structure.

${ }^{3}$ As general references on the geometry of nonholonomic manifolds and applications to modern physics, we cite $[24,30]$ and, for former constructions on tangent and vector bundles, [25, 15]. Readers may consult those works and provided there references and appendices for details and applications of the formalism of so-called dtensors and d-objects. We note that in this work similar constructions are generalized to the case when manifolds (in general, nonholonomic ones) are enabled with nonsymmetric metric structures and related linear connection structures.
} 
Definition 2.3. A d-metric $\check{g}_{i j}(x, y)$ is of index $k$ if there are satisfied the properties:

1) $\operatorname{det}\left|g_{i j}\right| \neq 0 \quad$ and

2) $\operatorname{rank}\left|a_{i j}\right|=n-k=2 p, \quad$ for $\quad 0 \leq k \leq n$.

We denote by $g^{i j}$ the reciprocal (inverse) to $g_{i j}$ d-tensor field. The matrix $\check{g}_{i j}$ is not invertible unless for $k=0$.

Definition 2.4. A N-anholonomic (we shall use also the term nonholonomic) Eisenhart space of index $k$ is a nonholonomic manifold $\left(\check{g}_{i j}, \mathbf{V}^{n+n}, \mathbf{N}\right)$ provided with d-metric $\check{g}_{i j}=g_{i j}+a_{i j}$ of index $k$.

For $k>0$ and a positive definite $g_{i j}(x, y)$, on each domain of local chart there exists $k$ d-vector fields $\xi_{i^{\prime}}^{i}$, where $i=1,2, \ldots, n$ and $i^{\prime}=1, \ldots, k$ with the properties

$$
a_{i j} \xi_{j^{\prime}}^{j}=0 \quad \text { and } \quad g_{i j} \xi_{i^{\prime}}^{i} \xi_{j^{\prime}}^{j}=\delta_{i^{\prime} j^{\prime}}
$$

If $g_{i j}$ is not positive definite, we shall assume the existence of $k$ linearly independent d-vector fields with such properties. For arbitrary signatures of $g_{i j}$, we can chose any $k$ independent and orthonormalized vectors defined as a linear combination of a linear basis of $n$ vectors.

We note that we can define completely the metric properties on $\mathbf{V}^{n+n}$ if we state additionally that this space is provided with the metric structure

$$
\begin{aligned}
& \check{\mathbf{g}}=\mathbf{g}+\mathbf{a}=\check{\mathbf{g}}_{\alpha \beta} \mathbf{e}^{\alpha} \otimes \mathbf{e}^{\beta}=\check{g}_{i j} e^{i} \otimes e^{j}+\check{g}_{a b} \mathbf{e}^{a} \otimes \mathbf{e}^{b}, \\
& \mathbf{g}=\mathbf{g}_{\alpha \beta} \mathbf{e}^{\alpha} \otimes \mathbf{e}^{\beta}=g_{i j} e^{i} \otimes e^{j}+g_{a b} \mathbf{e}^{a} \otimes \mathbf{e}^{b}, \\
& \mathbf{a}=a_{i j} e^{i} \wedge e^{j}+a_{c b} \mathbf{e}^{c} \wedge \mathbf{e}^{b},
\end{aligned}
$$

where the v-components $\check{g}_{a b}$ are defined by the same coefficients as $\check{g}_{i j}$.

Definition 2.5. A h-v-metric on a $\mathrm{N}$-anholonomic manifold is a second rank d-tensor of type (2.7).

We can define the local d-covector fields $\eta_{i}^{i^{\prime}}=g_{i j} \xi_{i^{\prime}}^{j}$ and the d-tensors of type $(1,1), l_{j}^{i}$ and $m_{j}^{i}$, satisfying the conditions

$$
\begin{aligned}
& l_{j}^{i}=\xi_{i^{\prime}}^{i} \eta_{j}^{i^{\prime}} \quad \text { and } \quad m_{j}^{i}=\delta_{j}^{i}-\xi_{i^{\prime}}^{i} \eta_{j}^{i^{\prime}}, \quad \text { for } \quad i^{\prime}=1, \ldots, k ; \\
& l_{j}^{i}=0 \quad \text { and } \quad m_{j}^{i}=\delta_{j}^{i}, \quad \text { for } \quad k=0 .
\end{aligned}
$$

For further computations, it is useful to use a matrix calculus. One denotes $A=\left(a_{i j}\right), B=\left(b^{i j}\right)$, or $C=\left(c_{j}^{i}\right)$, where the index $i$ specifies the row and the index $j$ specify the column. Thus $b^{i j} a_{j k}=c_{k}^{i}$ means $B A=C$ and $a_{i j} b^{j k}=c_{i}^{k}$ means $A B={ }^{t} C$, the transpose of $C$. We shall consider the matrices

$$
\begin{aligned}
& \widehat{g}=\left(g_{i j}\right), \quad \widehat{a}=\left(a_{i j}\right), \quad \widehat{\xi}=\left(\xi_{i^{\prime}}^{i}\right), \quad \widehat{l}=\left(l_{j}^{i}\right), \\
& \widehat{\eta}=\left(\eta_{i}^{i^{\prime}}\right), \quad \widehat{m}=\left(m_{j}^{i}\right), \quad \widehat{\delta}^{\prime}=\left(\delta_{i^{\prime} j^{\prime}}\right), \quad \widehat{\delta}=\left(\delta_{j}^{i}\right)
\end{aligned}
$$

for which the formulas

$$
\widehat{a} \widehat{\xi}=0, \quad{ }^{t} \widehat{\xi} \widehat{g} \widehat{\xi}=\widehat{\delta}, \quad{ }^{t} \widehat{\eta}=\widehat{g} \widehat{\xi}, \quad \widehat{l}=\widehat{\xi} \widehat{\eta}, \quad \widehat{m}=\widehat{\delta}-\widehat{l},
$$

imply

$$
\begin{array}{lll}
\widehat{a} \widehat{l}=0, & \widehat{a} \widehat{m}=\widehat{a}, \quad \widehat{\xi} \widehat{\eta}=\widehat{\delta}, \quad \widehat{\eta} \widehat{l}=\widehat{\eta}, & \widehat{\eta} \widehat{m}=0, \\
\widehat{g} \widehat{l}={ }^{t} \widehat{\eta \eta}, & \widehat{g} \widehat{m}=\widehat{g}-{ }^{t} \widehat{\eta} \widehat{\eta}, \quad \widehat{g} \widehat{l}^{-1}={ }^{t} \widehat{\xi} \widehat{\xi}, & \widehat{m} \widehat{g}^{-1}=\widehat{g}-{ }^{t} \widehat{\xi} \widehat{\xi} .
\end{array}
$$


One follows that the matrices $\widehat{g} \widehat{l}, \widehat{g} \widehat{m}, \widehat{l}^{-1}, \widehat{m} \widehat{g}^{-1}$ are symmetric. Similar matrices and formulas can be defined on the v-subspaces. They are labelled, for instance, $\widehat{g}=\left(g_{a b}\right), \widehat{a}=\left(a_{b c}\right), \widehat{\xi}=\left(\xi_{a^{\prime}}^{a}\right)$, $\widehat{l}=\left(l_{b}^{a}\right), \ldots$ We shall write ${ }^{h} \widehat{g}$ or ${ }^{v} \widehat{g}$ if it would be necessary to emphasize that a corresponding matrix is defined on h- or v-subspaces. For simplicity, we shall present only the formulas for the h-subspace and omit similar ones for the v-subspace.

Let us denote by ${ }^{d} \mathcal{X}(h \mathbf{V})$ the module of d-vector fields on $h \mathbf{V}$ (we note that $h \mathbf{V}=M$ if we take $\left.\mathbf{V}^{n+n}=T M\right)$. One considers the submodules

$$
\begin{aligned}
{ }^{h} \mathbf{K} & =\left\{\xi^{j} \in{ }^{d} \mathcal{X}\left(h \mathbf{V}^{n}\right) \mid a_{i j} \xi^{j}=0\right\}, \\
{ }^{h} \mathbf{H} & =\left\{\zeta^{i} \in{ }^{d} \mathcal{X}\left(h \mathbf{V}^{n}\right) \mid g_{i j} \xi^{i} \zeta^{j}=0, \forall \xi^{i} \in \mathbf{K}\right\} .
\end{aligned}
$$

The elements of ${ }^{h} \mathbf{K}$ are globally defined since they are in the kernel of the mapping $\xi^{j} \rightarrow a_{i j} \xi^{j}$. The structure ${ }^{h} \mathbf{H}$ is also global because its elements are orthogonal to ${ }^{h} \mathbf{K}$ which is locally spanned by $\left(\xi_{i^{\prime}}^{i}\right)$. One holds the following mutually equivalent conditions:

$$
\zeta^{i} \in{ }^{h} \mathbf{H}, \quad \eta_{i}^{i^{\prime}} \zeta^{i}=0, \quad l_{j}^{i} \zeta^{j}=0
$$

One also follows:

Proposition 2.1. The system of linear equations $a_{i j} X^{j}=0$ and $\eta_{i}^{i^{\prime}} X^{i}=0$ has only the trivial solution $X^{j}=0$.

The formulas (2.9) and (2.11) result in properties $l_{j}^{i} X^{j} \in{ }^{h} \mathbf{K}$ and $m_{j}^{i} X^{j} \in{ }^{h} \mathbf{H}$, for every $X^{j} \in d \mathcal{X}\left(h \mathbf{V}^{n}\right)$, and

$$
\widehat{l}+\widehat{m}=\widehat{\delta}, \quad \widehat{l}^{2}=\widehat{l}, \quad \widehat{m}^{2}=\widehat{m}, \quad \widehat{l} \widehat{m}=\widehat{m} \widehat{l}=0, \quad{ }^{t} \widehat{l} \widehat{g}=0,
$$

proving that the submodules ${ }^{h} \mathbf{K}$ and ${ }^{h} \mathbf{H}$ are orthogonal and supplementary, i.e. ${ }^{d} \mathcal{X}(h \mathbf{V})=$ ${ }^{h} \mathbf{K}+{ }^{h} \mathbf{H},{ }^{h} \mathbf{K} \cap{ }^{h} \mathbf{H}=\{\mathbf{0}\}$ and $g_{i j} \xi^{i} \zeta^{j}=0$, for every $\xi^{i} \in{ }^{h} \mathbf{K}$ and $\zeta^{j} \in{ }^{h} \mathbf{H}$. Following formulas (2.12), we conclude that $\widehat{l}$ and $\widehat{m}$ are unique projectors (tensor fields) not depending on $\widehat{\xi}$ which are completely determined, respectively, by ${ }^{h} \mathbf{K}$ and ${ }^{h} \mathbf{H}$ and globally defined on $\mathbf{V}^{n+n}$.

The next step is to extend the matrix $\widehat{a}$ to a nonsingular skew symmetric one of dimension $(n+k, n+k), \widetilde{a}=\left[\begin{array}{cc}\widehat{a} & -{ }^{t} \varphi \\ \varphi & 0\end{array}\right]$. The inverse matrix $\widetilde{a}^{-1}$, satisfying the condition $\widetilde{a} \widetilde{a}^{-1}=\widehat{\delta}$, has the form

$$
\widetilde{a}^{-1}=\left[\begin{array}{cc}
\check{a} & \widehat{\xi} \\
{ }^{t} \widehat{\xi} & 0
\end{array}\right],
$$

where the matrix $\check{a}=\left(\check{a}^{i j}\right)$ does not depend on the choice of $\widehat{\xi}$ and it is uniquely defined by $\widehat{a} \check{a}={ }^{t} \widehat{m}$ and $\widehat{l} \check{a}=0$, i.e. this matrix is uniquely defined on $\mathbf{V}^{n+n}$.

If the nonsymmetric part of metric vanishes, $\widehat{a}=0$, we have $\varphi=\widehat{\xi}^{-1}, \widehat{l}=\widehat{\delta}, \widehat{m}=0$ and $\check{a}=0$. In the case $k=0$, we have $\widehat{l}=0, \widehat{m}=\widehat{\delta}$ and $\check{a}=\widehat{a}^{-1}$ and if additionally $\widehat{g}=0$, the constructions reduces to an almost symplectic structure.

\section{Distinguished connections and nonsymmeric metrics}

We consider the basic properties of linear connections adapted to the N-connection structure on a nonholonomic manifold $\mathbf{V}^{n+n}$ enabled with nonsymmetric metric structure $\mathbf{g}$. 


\subsection{Torsion and curvature of d-connections}

In general, the concept of linear connection (adapted or not adapted to a N-connection structure) does not depend on the concept of metric (symmetric or nonsymmetric).

Definition 3.1. A distinguished connection (d-connection) $\mathbf{D}$ on $\mathbf{V}^{n+n}$ is a N-adapted linear connection, preserving by parallelism the vertical and horizontal distribution (2.1).

In local form, a d-connection $\mathbf{D}=\left({ }^{h} D,{ }^{v} D\right)$ is given by its coefficients $\boldsymbol{\Gamma}_{\alpha \beta}^{\gamma}=\left(L_{j k}^{i}, L_{b k}^{a}, C_{j c}^{i}\right.$, $\left.C_{b c}^{a}\right)$, where ${ }^{h} D=\left(L_{j k}^{i}, L_{b k}^{a}\right)$ and ${ }^{v} D=\left(C_{j c}^{i}, C_{b c}^{a}\right)$ are respectively the covariant h- and vderivatives.

The torsion $\mathcal{T}$ of a d-connection $\mathbf{D}$, is defined by the d-tensor field

$$
\mathcal{T}(\mathbf{X}, \mathbf{Y}) \doteqdot \mathbf{D}_{\mathbf{X}} \mathbf{Y}-\mathbf{D}_{\mathbf{Y}} \mathbf{X}-[\mathbf{X}, \mathbf{Y}]
$$

for any d-vectors $\mathbf{X}=h \mathbf{X}+v \mathbf{X}={ }^{h} \mathbf{X}+{ }^{v} \mathbf{X}$ and $\mathbf{Y}=h \mathbf{Y}+v \mathbf{Y}$, with a corresponding N-adapted decomposition into

$$
\begin{aligned}
& \mathcal{T}(\mathbf{X}, \mathbf{Y})=\{h \mathcal{T}(h \mathbf{X}, h \mathbf{Y}), h \mathcal{T}(h \mathbf{X}, v \mathbf{Y}), h \mathcal{T}(v \mathbf{X}, h \mathbf{Y}), h \mathcal{T}(v \mathbf{X}, v \mathbf{Y}), \\
&v \mathcal{T}(h \mathbf{X}, h \mathbf{Y}), v \mathcal{T}(h \mathbf{X}, v \mathbf{Y}), v \mathcal{T}(v \mathbf{X}, h \mathbf{Y}), v \mathcal{T} v \mathbf{X}, v \mathbf{Y})\}
\end{aligned}
$$

The nontrivial N-adapted coefficients of

$$
\mathcal{T}=\left\{\mathbf{T}_{\beta \gamma}^{\alpha}=-\mathbf{T}_{\gamma \beta}^{\alpha}=\left(T_{j k}^{i}, T_{j a}^{i}, T_{j k}^{a}, T_{j a}^{b}, T_{c a}^{b}\right)\right\}
$$

can be computed by introducing $\mathbf{X}=\mathbf{e}_{\alpha}$ and $\mathbf{Y}=\mathbf{e}_{\beta}$ into (3.1), see formulas (A.1) in Appendix and [24].

The curvature of a d-connection $\mathbf{D}$ is defined

$$
\mathcal{R}(\mathbf{X}, \mathbf{Y}) \doteqdot \mathbf{D}_{\mathbf{X}} \mathbf{D}_{\mathbf{Y}}-\mathbf{D}_{\mathbf{Y}} \mathbf{D}_{\mathbf{X}}-\mathbf{D}_{[\mathbf{X}, \mathbf{Y}]},
$$

with N-adapted decomposition

$$
\begin{aligned}
\mathcal{R}(\mathbf{X}, \mathbf{Y}) \mathbf{Z}= & \{\mathcal{R}(h \mathbf{X}, h \mathbf{Y}) h \mathbf{Z}, \mathcal{R}(h \mathbf{X}, v \mathbf{Y}) h \mathbf{Z}, \mathcal{R}(v \mathbf{X}, h \mathbf{Y}) h \mathbf{Z}, \mathcal{R}(v \mathbf{X}, v \mathbf{Y}) h \mathbf{Z} \\
& \mathcal{R}(h \mathbf{X}, h \mathbf{Y}) v \mathbf{Z}, \mathcal{R}(h \mathbf{X}, v \mathbf{Y}) v \mathbf{Z}, \mathcal{R}(v \mathbf{X}, h \mathbf{Y}) v \mathbf{Z}, \mathcal{R}(v \mathbf{X}, v \mathbf{Y}) v \mathbf{Z}\}
\end{aligned}
$$

The formulas for local N-adapted components and their symmetries, of the d-torsion and dcurvature, can be computed by introducing $\mathbf{X}=\mathbf{e}_{\alpha}, \mathbf{Y}=\mathbf{e}_{\beta}$ and $\mathbf{Z}=\mathbf{e}_{\gamma}$ in (3.2). The nontrivial $\mathrm{N}$-adapted coefficients

$$
\mathcal{R}=\left\{\mathbf{R}_{\beta \gamma \delta}^{\alpha}=\left(R_{h j k}^{i}, R_{b j k}^{a}, R_{h j a}^{i}, R_{b j a}^{c}, R_{h b a}^{i}, R_{b e a}^{c}\right)\right\}
$$

are given by formulas (A.2) in Appendix, see also [24].

Contracting the first and forth indices $\mathbf{R}_{\beta \gamma}=\mathbf{R}_{\beta \gamma \alpha}^{\alpha}$, one gets the N-adapted coefficients for the Ricci tensor

$$
\text { Ric } \doteqdot\left\{\mathbf{R}_{\beta \gamma}=\left(R_{i j}, R_{i a}, R_{a i}, R_{a b}\right)\right\},
$$

see formulas (A.3) in Appendix and [24]. It should be noted here that for general d-connections the Ricci tensor is not symmetric, i.e. $\mathbf{R}_{\beta \gamma} \neq \mathbf{R}_{\gamma \beta}$.

On spaces of dimension $n+n$, it is convenient to work with a particular class of d-connections.

Definition 3.2. A normal d-connection ${ }_{n} \mathbf{D}$ is compatible with the almost complex structure $\mathbb{F}(2.6)$, i.e. satisfies the condition

$$
{ }_{n} \mathbf{D}_{\mathbf{X}} \mathbb{F}=0,
$$

for any d-vector $\mathbf{X}$ on $\mathbf{V}^{n+n}$. 
From local formulas for (3.3), one follows:

Theorem 3.1. A normal d-connection is characterized by a pair of local coefficients ${ }_{n} \boldsymbol{\Gamma}_{\alpha \beta}^{\gamma}=$ $\left({ }_{n} L_{j k}^{i},{ }_{n} C_{b c}^{a}\right)$ defined by conditions

$$
\begin{aligned}
& { }_{n} \mathbf{D}_{\mathbf{e}_{k}}\left(\mathbf{e}_{j}\right)={ }_{n} L_{j k}^{i} \mathbf{e}_{i}, \quad{ }_{n} \mathbf{D}_{\mathbf{e}_{k}}\left(e_{a}\right)={ }_{n} L_{a k}^{b} e_{b}: \quad \text { for } \quad j=a, i=b, \quad{ }_{n} L_{j k}^{i}={ }_{n} L_{a k}^{b}, \\
& { }_{n} \mathbf{D}_{e_{c}}\left(\mathbf{e}_{j}\right)={ }_{n} C_{j c}^{i} \mathbf{e}_{i}, \quad{ }_{n} \mathbf{D}_{e_{c}}\left(e_{a}\right)={ }_{n} C_{a c}^{b} e_{b}: \quad \text { for } \quad j=a, i=b, \quad{ }_{n} C_{j c}^{i}={ }_{n} C_{a c}^{b} .
\end{aligned}
$$

By a straightforward local calculus introducing the pairs of coefficients for $_{n} \boldsymbol{\Gamma}_{\alpha \beta}^{\gamma}$, respectively, in (3.1) and (3.2), see also formulas (A.1) and (A.2) in Appendix, we prove

Corollary 3.1. The $N$-adapted coefficients of torsion ${ }_{n} \mathcal{T}=\left\{{ }_{n} T_{\beta \gamma}^{\alpha}\right\}$ and curvature ${ }_{n} \mathcal{R}=$ $\left\{{ }_{n} R_{\beta \gamma \tau}^{\alpha}\right\}$ of the normal d-connection ${ }_{n} \mathbf{D}$ are defined respectively by formulas

$$
\begin{aligned}
& { }_{n} T^{i}{ }_{j k}={ }_{n} L^{i}{ }_{j k}-{ }_{n} L^{i}{ }_{k j}, \\
& { }_{n} T_{b i}^{a}=\frac{\partial N_{i}^{a}}{\partial y^{b}}-{ }_{n} L_{b i}^{a},
\end{aligned}
$$

and

$$
\begin{aligned}
& { }_{n} R_{h j k}^{i}=\mathbf{e}_{k}\left({ }_{n} L^{i}{ }_{h j}\right)-\mathbf{e}_{j}\left({ }_{n} L^{i}{ }_{h k}\right)+{ }_{n} L_{h j n}^{m} L^{i}{ }_{m k}-{ }_{n} L_{h k n}^{m} L^{i}{ }_{m j}-{ }_{n} C^{i}{ }_{h a} \Omega^{a}{ }_{k j}, \\
& { }_{n} R_{b k a}^{c}=e_{a}\left({ }_{n} L_{b k}^{c}\right)-{ }_{n} \mathbf{D}_{k}\left({ }_{n} C_{b a}^{c}\right)+{ }_{n} C_{b d n}^{c} T_{k a}^{c} \text {, } \\
& { }_{n} R_{b c d}^{a}=e_{d}\left({ }_{n} C^{a}{ }_{b c}\right)-e_{c}\left({ }_{n} C^{a}{ }_{b d}\right)+{ }_{n} C_{b c n}^{e} C^{a}{ }_{e d}-{ }_{n} C^{e}{ }_{b d n} C^{a}{ }_{e c} .
\end{aligned}
$$

In the geometry of $\mathrm{N}$-anholonomic manifolds and Finsler-Lagrange spaces an important role is given to a special type of d-connections:

Definition 3.3. A normal d-connection ${ }_{c} \boldsymbol{\Gamma}_{\alpha \beta}^{\gamma}=\left({ }_{c} L_{j k}^{i},{ }_{c} C_{b c}^{a}\right)$ on $\mathbf{V}^{n+n}$ is a Cartan d-connection if it satisfies the conditions

$$
{ }_{c}^{h} D_{k} y^{a}=0 \quad \text { and } \quad{ }_{c}^{v} D_{a} y^{b}=\delta_{a}^{b} .
$$

By an explicit local N-adapted calculus, we can verify:

Proposition 3.1. The $N$-connection and Cartan d-connection coefficients satisfy the conditions

$$
N_{i}^{a}=y^{b}{ }_{c} L_{b i}^{a} \quad \text { and } \quad y^{b}{ }_{c} C_{b c}^{a}=0
$$

and the $d$-torsions and $d$-curvatures are related by formulas

$$
{ }_{c} T_{k j}^{a}=\Omega^{a}{ }_{k j}=y^{b}{ }_{c} R_{b j k}^{a}, \quad{ }_{c} T_{b i}^{c}=y^{a}{ }_{c} R^{c}{ }_{a k b}, \quad{ }_{c} T_{b c}^{a}=y^{d}{ }_{c} R_{d b c}^{a} .
$$

Finally, we note that on $\mathrm{N}$-anholonomic manifolds we can work equivalently with different classes of d-connections. For applications in modern physics [24, 30, 17, 23], the constructions with metric compatible d-connections which are defined in a unique way by a metric and certain prescribed torsion structures are considered to be more related to standard theories.

\subsection{Distinguished connections compatible with nonsymmetric metrics}

In this section, we define a class of d-connections which are compatible with nonsymmetric metric structures.

Let us consider a nonholonomic manifold $\mathbf{V}^{n+n}$ with fixed $\mathrm{N}$-connection $\mathbf{N}$ and enabled with a d-connection $\mathbf{D}_{\alpha}=\left(D_{i}, D_{a}\right)$ and nonsymmetric metric structure $\check{\mathbf{g}}=\mathbf{g}+\mathbf{a}$. A nonsymmetric metric $\check{\mathrm{g}}$ is characterized by d-tensor fields/matrices (2.8) satisfying the properties (2.9) and (2.12). Considering actions of a general d-connection $\mathbf{D}$ on the mentioned formulas, one prove: 
Proposition 3.2. One holds the following formulas for the h-covariant (v-covariant) derivatives of the coefficients of matrices (2.8) defined globally by a nonsymmetric metric structure:

$$
\begin{array}{lc}
l_{p}^{i} l_{j}^{q} D_{k} l_{q}^{p}=0, \quad m_{p}^{i} D_{k} l_{q}^{p}=l_{q}^{s} D_{k} l_{s}^{i}, \quad m_{j}^{s} D_{k} l_{s}^{i}=l_{q}^{i} D_{k} l_{j}^{q}, \\
l_{i}^{p} l_{j}^{q} D_{k} a_{p q}=0, \quad a_{s j} \check{a}^{i r} D_{k} l_{r}^{s}=0, \quad l_{j}^{s}\left(\check{a}^{i r} D_{k} a_{r s}-D_{k} l_{s}^{i}\right)=0
\end{array}
$$

(one v-subspace the formulas are similar but with ${ }^{h} D=\left\{D_{k}\right\} \rightarrow{ }^{v} D=\left\{D_{a}\right\}$ ).

Proof. In order to prove the formulas (3.5), it is convenient to use the matrix covariant hderivative $D_{k} \widehat{l}=\left(D_{k} l_{j}^{i}\right)$ and v-derivative $D_{a} \widehat{l}=\left(D_{a} l_{j}^{i}\right)$ which for $\widehat{l}^{2}=\widehat{l}$ imply respectively $D_{k} \widehat{l l}+\widehat{l} D_{k} \widehat{l}=\widehat{l}$ and $D_{a} \widehat{l l}+\widehat{l} D_{a} \widehat{l}=\widehat{l}$. One should be used the matrix relation $\widehat{a} \widehat{l}=0$ to get the formulas containing the anti-symmetric part of metric.

We shall work with a more restricted class of d-connections on $\mathbf{V}^{n+n}$ :

Definition 3.4. A d-connection $\mathbf{D}=\left\{\boldsymbol{\Gamma}_{\alpha \beta}^{\gamma}=\left(L_{j k}^{i}, C_{b c}^{a}\right)\right\}$ is compatible with a nonsymmetric d-metric $\check{g}$ if

$$
\mathbf{D}_{k} \check{g}_{i j}=0 \quad \text { and } \quad \mathbf{D}_{a} \check{g}_{i j}=0 .
$$

For the d-metric (2.7), the equations (3.6) are written $\mathbf{D}_{k} g_{i j}=0, \mathbf{D}_{a} g_{b c}=0, \mathbf{D}_{k} a_{i j}=0$, $\mathbf{D}_{e} a_{b c}=0$.

In such cases, there are additional to (3.5) properties:

Proposition 3.3. The d-tensor fields $\widehat{l}=\left(l_{j}^{i}\right), \widehat{m}=\left(m_{j}^{i}\right), \check{g}=\left(g^{i j}\right)$ and $\check{a}=\left(\check{a}^{i j}\right)$ satisfy the conditions

$$
\begin{array}{rlrlrl}
D_{k} l_{j}^{i} & =0, & D_{k} m_{j}^{i}=0, & D_{k} g^{i j}=0, & D_{k} \check{a}^{i j}=0, \\
D_{a} l_{c}^{b}=0, & D_{a} m_{c}^{b}=0, & D_{a} g^{b c}=0, & D_{a} \check{a}^{b c}=0,
\end{array}
$$

for any $\mathbf{D}$ compatible to $\check{\mathbf{g}}$.

Proof. It is important to take the covariant h- and v-derivatives for formulas $a_{i j} \xi_{i^{\prime}}^{i}=0$, $g_{i j} \xi_{i^{\prime}}^{i} \xi_{j^{\prime}}^{j}=\delta_{i^{\prime} j^{\prime}}, a_{r s} \check{a}^{s j}=m_{r}^{j}$ and $l_{s}^{i} \check{a}^{s j}=0$ and use the properties (2.9) and (2.12).

It is important to define the geometric properties of tensor fields from Proposition 3.3 because they are used to formulate and prove main Theorems 5.3 and 5.4.

To work with metric compatible connections is not only a preferred approach in order to elaborate more "simple" physical theories, but it is motivated geometrically by the fact that there is a method of metrization for arbitrary d-connections.

\section{4 (Non)symmetric metrization procedure of d-connections}

Usually, in gravity theories, one fix a linear connection connection structure which is, or not, metric compatible and, in general, with nontrivial torsion (in general relativity, this is the LeviCivita connection which by definition is both metric compatible and torsionless). In Finsler geometry, A. Kawaguchi [32, 33, 34] proposed the method of metrization of d-connections which was further developed for Lagrange spaces, see [25, 15]. The approach was also used for Einstein and Riemann-Cartan spaces defined by generic off-diagonal metrics when the nonholonomic deformations were considered not only for frame and metric structures but also for linear connections in order to generate such ansatz for geometric objects when the field equations became exactly integrable for certain systems on nonlinear partial differential equations, see review [24]. 
It was emphasized that imposing additional constraints on integral varieties of solutions for more general classes of connections it is possible to generate solutions for the Levi-Civita connection. In this section, we show how the metrization method can be applied on $\mathrm{N}$-anholonomic manifolds enabled with nonsymmetric metrics.

A fixed metric structure on a nonholonomic manifold induces certain (Obata) type operators defining the set of d-connections being compatible with this metric structure. The aim of this section is to define the properties of such operators.

\subsection{Metrization methods with symmetric metrics}

By straightforward computations, for symmetric metrics on vector bundles, one proved two important results which hold true for N-anholonomic manifolds:

1. Kawaguchi's metrization: To any fixed d-connection ${ }_{\circ} \mathbf{D}$ we can associate a compatible with a metric $\mathbf{g}$ d-connection $\mathbf{D}$, satisfying the condition $\mathbf{D}_{\mathbf{X}} \mathbf{g}=\mathbf{0}$ for any d-vector on $\mathbf{V}^{n+n}$. The N-adapted coefficients for d-connections are related by formulas

$$
\begin{array}{ll}
L_{j k}^{i}={ }_{\circ} L_{j k}^{i}+\frac{1}{2} g^{i m}{ }_{\circ} D_{k} g_{m j}, & L_{b k}^{a}={ }_{\circ} L_{b k}^{a}+\frac{1}{2} g^{a c}{ }_{\circ} D_{k} g_{c b}, \\
C^{i}{ }_{j c}={ }_{\circ} C_{j c}^{i}+\frac{1}{2} g^{i m}{ }_{\circ} D_{c} g_{m j}, & C_{b c}^{a}={ }_{\circ} L_{b c}^{a}+\frac{1}{2} g^{a e}{ }_{\circ} D_{c} g_{e b} .
\end{array}
$$

2. Miron's procedure: The set of d-connections $\{\mathbf{D}\}$ satisfying the conditions $\mathbf{D}_{\mathbf{X}} \mathbf{g}=\mathbf{0}$ for a given $\mathbf{g}$ is defined by formulas

$$
\begin{array}{ll}
L^{i}{ }_{j k}=\widehat{L}_{j k}^{i}+{ }^{-} O_{k m}^{e i} \mathbf{X}_{e j}^{m}, & L_{b k}^{a}=\widehat{L}_{b k}^{a}+{ }^{-} O_{b d}^{c a} \mathbf{Y}_{c k}^{d}, \\
C^{i}{ }_{j c}=\widehat{C}_{j c}^{i}+{ }^{+} O_{j k}^{m i} \mathbf{X}_{m c}^{k}, & C^{a}{ }_{b c}=\widehat{C}_{b c}^{a}+{ }^{+} O_{b d}^{e a} \mathbf{Y}_{e c}^{d},
\end{array}
$$

where

$$
{ }^{ \pm} O_{j k}^{i h}=\frac{1}{2}\left(\delta_{j}^{i} \delta_{k}^{h} \pm g_{j k} g^{i h}\right), \quad{ }^{ \pm} O_{b d}^{c a}=\frac{1}{2}\left(\delta_{b}^{c} \delta_{d}^{a} \pm g_{b d} g^{c a}\right)
$$

are the so-called the Obata operators; $\mathbf{X}_{e j}^{m}, \mathbf{X}_{m c}^{k}, \mathbf{Y}_{c k}^{d}$ and $\mathbf{Y}_{e c}^{d}$ are arbitrary d-tensor fields and $\widehat{\boldsymbol{\Gamma}}_{\alpha \beta}^{\gamma}=\left(\widehat{L}_{j k}^{i}, \widehat{L}_{b k}^{a}, \widehat{C}_{j c}^{i}, \widehat{C}_{b c}^{a}\right)$, with

$$
\begin{aligned}
& \widehat{L}_{j k}^{i}=\frac{1}{2} g^{i r}\left(e_{k} g_{j r}+e_{j} g_{k r}-e_{r} g_{j k}\right), \\
& \widehat{L}_{b k}^{a}=e_{b}\left(N_{k}^{a}\right)+\frac{1}{2} g^{a c}\left(e_{k} g_{b c}-g_{d c} e_{b} N_{k}^{d}-g_{d b} e_{c} N_{k}^{d}\right), \\
& \widehat{C}_{j c}^{i}=\frac{1}{2} g^{i k} e_{c} g_{j k}, \quad \widehat{C}_{b c}^{a}=\frac{1}{2} g^{a d}\left(e_{c} g_{b d}+e_{c} g_{c d}-e_{d} g_{b c}\right)
\end{aligned}
$$

is the canonical d-connections uniquely defined by the coefficients of d-metric $\mathbf{g}=\left[g_{i j}, g_{a b}\right]$ and N-connection $\mathbf{N}=\left\{N_{i}^{a}\right\}$ in order to satisfy the conditions $\widehat{\mathbf{D}}_{\mathbf{X}} \mathbf{g}=\mathbf{0}$ and $\widehat{T}^{i}{ }_{j k}=0$ and $\widehat{T}_{b c}^{a}=0$ but $\widehat{T}_{j a}^{i}, \widehat{T}_{j i}^{a}$ and $\widehat{T}_{b i}^{a}$ are not zero, see formulas (A.1). On $\mathbf{V}^{n+n}$, it is possible to work with the normal, or the Cartan, d-connection as we defined by (3.3), or (3.4).

\subsection{Properties of Obata operators for nonsymmetric d-metrics}

The A. Kawaguchi and R. Miron metrization procedures can be generalized for nonsymmetric metrics $\check{\mathbf{g}}=\mathbf{g}+\mathbf{a}(2.7)$ on $\mathrm{N}$-anholonomic manifolds. For simplicity, we consider the formulas for h-subspaces of a $\mathbf{V}^{n+n}$ with fixed $\mathrm{N}$-connection structure. 
On h-subspace, for the symmetric part $\mathbf{g}$ of $\check{\mathbf{g}}$, the Obata operators are of type ${ }_{h}^{[a]} O=\left\{{ }^{ \pm} O_{j k}^{i h}\right\}$ (4.1), for $[a]= \pm$. In general, we have on $\mathbf{V}^{n+n}$ the operators $\mathbf{O}=\left({ }_{h}^{[a]} O,{ }_{v}^{[a]} O\right)$. These operators act on d-tensor fields of type $(1,2)$ following the $\operatorname{rule}\left({ }_{h}^{ \pm} O X\right)_{j k}^{i}={ }^{ \pm} O_{s j}^{i h} \mathbf{X}_{h k}^{s}$, when the product of Obata h-operators, ${ }_{h}^{[a]} \mathrm{O}_{h}^{[b]} \mathrm{O}$, is defined

$$
\left({ }_{h}^{[a]} O_{h}^{[b]} O\right)_{s j}^{i m}={ }_{h}^{[a]} O_{k j h}^{i r}{ }^{[b]} O_{s r}^{k m} .
$$

We can check by explicit computations:

Proposition 4.1. There are satisfied the properties

$$
{ }_{h}^{-} O+{ }_{h}^{+} O=I, \quad\left({ }_{h}^{[a]} O\right)^{2}={ }_{h}^{[a]} O, \quad{ }_{h}^{-} O_{h}^{+} O={ }_{h}^{+} O_{h}^{-} O=0,
$$

where $I$ is the identity matrix $\delta_{s}^{i} \delta_{j}^{r}$, when $I \mathbf{X}=\mathbf{X}$.

This Proposition states that the operators $\mathbf{O}$ are supplementary projectors on the module of d-tensor fields of type $(1,2)$.

The skewsymmetric part a of $\check{\mathbf{g}}$ defines an additional set of Obata operators $\boldsymbol{\Phi}=\left({ }_{h}^{[a]} \Phi,{ }_{v}^{[a]} \Phi\right)$, where, for instance, $2\left({ }_{h}^{ \pm} \Phi\right)_{s j}^{i r}=\delta_{s}^{i} \delta_{j}^{r} \pm\left(l_{s}^{i} l_{j}^{r}+a_{s j} \check{a}^{i r}\right)$.

Introducing the operator ${ }_{h} \theta=\left\{\theta_{s j}^{i r}\right\}$, with $\theta_{s j}^{i r}=\frac{1}{2}\left(l_{s}^{i} m_{j}^{r}+m_{s}^{i} l_{j}^{r}\right)$, one proves by algebraic computations:

Proposition 4.2. One holds the relations

$$
{ }_{h}^{-} \Phi+{ }_{h}^{+} \Phi=I, \quad\left({ }_{h}^{[a]} \Phi\right)^{2}={ }_{h}^{[a]} \Phi-\frac{1}{2} h \theta, \quad{ }_{h}^{-} \Phi_{h}^{+} \Phi={ }_{h}^{+} \Phi_{h}^{-} \Phi=\frac{1}{2}{ }_{h} \theta .
$$

The relations for "skewsymmetric" Obata operators (4.4) are different from those for the symmetric ones (4.3). We can modify the constructions by introducing $\boldsymbol{\Psi}=\left({ }_{h}^{[a]} \Psi,{ }_{v}{ }_{v}^{[a]} \Psi\right)$, where, for instance, ${ }_{h}^{ \pm} \Psi={ }_{h}^{ \pm} \Phi \pm{ }_{h} \theta$.

By direct computations, one proves

Proposition 4.3. The operators $\boldsymbol{\Psi}$ are supplementary projectors on the module of d-tensor fields of type $(1,2)$ and satisfy the conditions (for simplicity, we state them for the h-subspace):

$$
\begin{array}{lll}
{ }_{h}^{-} \Psi+{ }_{h}^{+} \Psi=I, & \left({ }_{h}^{[a]} \Psi\right)^{2}={ }_{h}^{[a]} \Psi, \quad{ }_{h} \Psi_{h}^{+} \Psi={ }_{h}^{+} \Psi_{h}^{-} \Psi=0, & l_{s}^{i} a_{s j}\left({ }_{h}^{ \pm} \Psi\right)_{p r}^{s m}=0, \\
l_{s}^{i} m_{j}^{r}\left({ }_{h}^{ \pm} \Psi\right)_{p r}^{s m}=0, & { }_{h}^{+} \Psi_{h} \theta={ }_{h} \theta_{h}^{+} \Psi, & { }_{h} \Psi_{h} \theta={ }_{h} \theta_{h}^{-} \Psi={ }_{h} \theta .
\end{array}
$$

One follows from properties (4.4) and (4.5) that the operators ${ }_{h}^{[a]} \Phi$ and ${ }_{h}^{[a]} \Psi$ commute with $\theta$.

Finally, we note that even a number of properties of Obata operators ${ }_{h}^{[a]} \Psi$ (or ${ }_{h}^{[a]} \Phi$ ), for the skewsymmetric part $\mathbf{a}$ of a nonsymmetric metric $\check{\mathbf{g}}=\mathbf{g}+\mathbf{a}$ are similar to the properties of Obata operators ${ }_{h}^{[a]} O$ for the symmetric part $\mathbf{g}$, there is a substantial difference between these two classes of projectors. If $\theta$ commutes with ${ }_{h}^{[a]} O$, the operators ${ }_{h}^{[a]} \Phi$ and ${ }_{h}^{[a]} \Psi$ do not necessarily commute with ${ }_{h}^{[a]} O$. For instance, we have

$$
\left({ }_{h}^{+} O_{h}^{+} \Phi-{ }_{h}^{+} \Phi_{h}^{+} O\right)_{p j}^{i m}=\frac{1}{4}\left(g_{s j} g^{i r} a_{p r} \check{a}^{s m}-a_{s j} \check{a}^{i r} g_{p r} g^{s m}\right) .
$$

We need some additional suppositions on commutations of symmetric and skewsymmetric Obata operators imposing certain nonholonomic constraints on the components of nonsymmetric metrics. 


\subsection{Natural nonsymmetric metrics}

Let us define a subclass of nonsymmetric d-metrics for which the procedure of metrization of d-connections will be commutative both for the symmetric and skewsymmetric parts.

Definition 4.1. A nonsymmetric d-metric $\check{\mathbf{g}}=\mathbf{g}+\mathbf{a}$ is natural (2.7) if its associated symmetric and skewsymmetric Obata operators satisfy at least one of the conditions (for simplicity, we state them for the h-part)

$$
{ }_{h}^{[a]} O_{h}^{[b]} \Phi={ }_{h}^{[a]} \Phi_{h}^{[b]} O \quad \text { and } \quad{ }_{h}^{[a]} O_{h}^{[b]} \Psi={ }_{h}^{[a]} \Psi_{h}^{[b]} O .
$$

We emphasize that, if one of the commutation rules holds true from the set of eight ones (4.7), the statement is true for all projectors. So, we can verify the commutation rule only for any two operators and consider that similar commutation rules exist for other operators.

Let us consider a set of unknown d-tensor of type $(1,2)$ denoted by symbols $\mathbf{Y}, \mathbf{U}, \mathbf{V}, \mathbf{W}$ which are supposed to be connected to actions of Obata operators on $\mathbf{X}$ being also a d-tensor of type $(1,2)$. One holds:

Proposition 4.4. The equation ${ }_{h}^{-} O \mathbf{X}=0$ has the general solution $\mathbf{X}={ }_{h}^{+} O \mathbf{Y}$, where $\mathbf{Y}$ is arbitrary. There are also mutually equivalent the equations ${ }_{h}^{-} \Phi \mathbf{X}=\mathbf{W}$ and ${ }_{h}^{-} \Psi \mathbf{X}=\mathbf{W}+2{ }_{h} \theta \mathbf{W}$.

Proof. The statement on general solution is an obvious conclusion from the properties of matrix equations. The statement on equivalence of two matrix equations follows from the fact that multiplying those equations on $\theta$ one gets $\theta \mathbf{X}=2 \theta \mathbf{W}$ which proves the equivalence.

For the Obata operators defining the class of natural nonsymmetric d-metrics, we provide:

Theorem 4.1. Let us suppose that ${ }_{h}^{+} O_{h}^{+} \Psi={ }_{h}^{+} \Psi_{h}^{+} O$ and consider the system of equations

$$
{ }_{h}^{-} O \mathbf{X}=\mathbf{U}, \quad{ }_{h}^{+} \Psi \mathbf{X}=\mathbf{V}
$$

has solutions if and only if

$$
{ }_{h}^{+} O \mathbf{U}=0, \quad{ }_{h}^{+} \Psi \mathbf{V}=0, \quad{ }_{h}^{-} \Psi \mathbf{U}={ }_{h}^{-} O \mathbf{V} .
$$

The general solutions (4.8) can be written in two equivalent forms

$$
\mathbf{X}=\mathbf{U}+{ }_{h}^{+} O\left(\mathbf{V}+{ }_{h}^{+} \Psi \mathbf{Y}\right)=\mathbf{V}+{ }_{h}^{+} \Psi\left(\mathbf{U}+{ }_{h}^{+} O \mathbf{Y}\right)
$$

for arbitrary d-tensors $\mathbf{Y}$.

Proof. The necessity conditions follow from Propositions 4.1 and 4.3 and conditions (4.7). To prove the enough conditions we assume that the conditions (4.9) are satisfied. The first equation in (4.8) is equivalent to ${ }_{h}^{-} O(\mathbf{X}-\mathbf{U})=0$ having the general solution $\mathbf{X}=\mathbf{U}+{ }_{h}^{+} O \mathbf{Y}$, with arbitrary $\mathbf{Y}$, which solves also the equation ${ }_{h}^{-} \Psi \mathbf{X}=\mathbf{V}$ if and only if ${ }_{h}^{-} \Psi\left(\mathbf{U}+{ }_{h}^{+} O \mathbf{Y}\right)=\mathbf{V}$. We have ${ }_{h}^{-} \Psi=I-{ }_{h}^{+} \Psi$ which allows to write $\mathbf{U}+{ }_{h}^{+} O \mathbf{Y}=\mathbf{V}+{ }_{h}^{+} \Psi\left(U+{ }_{h}^{+} O \mathbf{Y}\right)$ proving that the d-tensor $\mathbf{X}$ can be represented in the forms (4.10) when obviously both forms solve the equations (4.8).

We can formulate a criteria of existence of normal nonsymmetric d-metrics:

Theorem 4.2. A nonsymmetric d-metric $\check{g}_{i j}(x, y)$ on $N$-anholonomic manifold $\mathbf{V}^{n+n}$ is natural if and only if one exists a non-vanishing real scalar function $\nu(x, y)$ such that

$$
\check{a}_{i j}=\nu a_{i j}
$$

where $a_{i j}=g_{i r} g_{j s} \check{a}^{r s}$. 
Proof. Taking from the conditions (4.7) the relation ${ }_{h}^{+} O_{h}^{+} \Psi={ }_{h}^{+} \Psi_{h}^{+} O$, we express (4.6), multiplied on $g_{i m} g_{t h}$, in the form

$$
\check{a}_{i j} a_{k l}=\check{a}_{k l} a_{i j} .
$$

Contracting this expression by $\check{a}^{l k}$ together with $a_{l k} \check{a}^{l k}=m_{k}^{k}=n-k \neq 0$, we get (4.11) with $\nu=\left(\check{a}_{l k} \check{a}^{l k}\right) / n-k \neq 0$ because otherwise we get $\check{a}_{l k}=0$ and $\check{a}^{l k}=0$ imposing $m_{j}^{i}=0$ which contradicts $k<n$. Conversely, the equation (4.11) with $\nu \neq 0$ transforms (4.12) into ${ }_{h}^{+} O_{h}^{+} \Psi={ }_{h}^{+} \Psi_{h}^{+} O$.

Two examples how to construct natural nonsymmetric metrics in explicit form will be provided below in Section 5.1. Finally, we emphasize that the metrization method proposed in this section is more particular than the Kawaguchi and Miron methods outlined in [25], although it is in a more general setting of Eisenhart N-anholonomic manifolds.

\section{5 d-connections compatible with nonsymmetric metrics}

For any natural nonsymmetric d-metric, we can define in explicit form the set of d-connections compatible to this metric structure. The goal of this section is to provide explicit methods of constructing natural nonsymmetric d-metrics and elaborate a method for metrization of arbitrary d-connections, with respect to such a d-metric.

\subsection{Examples of natural nonsymmetric metrics}

Let us consider two quadruplets $\left(g_{i j},{ }_{ \pm} F_{j}^{i}, \xi_{i^{\prime}}^{j}, \eta_{i}^{i^{\prime}}\right)$, where $g_{i j}$ is a symmetric d-metric; ${ }_{+} \widehat{F}=$ $\left({ }_{+} F_{j}^{i}\right)$ and ${ }_{-} \widehat{F}=\left({ }_{-} F_{j}^{i}\right)$ are d-tensor fields; $\xi_{i^{\prime}}^{j}$ are $k$ d-covector fields and $\eta_{i}^{i^{\prime}}=g_{i j} \xi_{i^{\prime}}^{j}$, where $i, j, \ldots=1,2, \ldots, n$ and $i^{\prime}, j^{\prime}, \ldots=1,2, \ldots, k$.

Definition 5.1. A respective quadruplet defines a $\left(\widehat{g},{ }_{ \pm} \widehat{F}, \widehat{\xi}, \widehat{\eta}\right)$-structure of index $k$ if one holds the conditions

$$
{ }_{ \pm} \widehat{F}^{2}=\mp \widehat{\delta} \pm \widehat{\xi} \widehat{\eta}, \quad \widehat{\eta}_{ \pm} \widehat{F}=0, \quad{ }_{ \pm} \widehat{F} \widehat{\xi}=0, \quad \widehat{\eta} \widehat{\xi}=\widehat{\delta}, \quad{ }_{ \pm}^{t} \widehat{F} \widehat{g}_{ \pm} \widehat{F}= \pm \widehat{g} \mp{ }^{t} \widehat{\eta} \widehat{\eta} .
$$

For $k>0$, one holds $\left({ }_{ \pm} \widehat{F}\right)^{3}={ }_{ \pm} \widehat{F}$. One assumes that for $k=0$ the conditions (5.1) transform into ${ }_{ \pm} \widehat{F}^{2}=\mp \widehat{\delta}$ and ${ }_{ \pm}^{t} \widehat{F} \widehat{g}_{ \pm} \widehat{F}= \pm \widehat{g}$ and define an almost Hermitian structure for ${ }_{+} \widehat{F}$, or an almost hyperbolic structure for $-\widehat{F}$. In the hyperbolic case, there is an eigen d-vector $v$ such that ${ }_{-} \widehat{F} Z= \pm Z$, when ${ }_{-}^{t} \widehat{F} \widehat{g}_{-} \widehat{F}=-\widehat{g}$ leads to ${ }^{t} Z \widehat{g} Z=0$, which holds for a nonpositively defined symmetric metric $\widehat{g}$.

Theorem 5.1. We obtain a natural nonsymmetric d-metric $\check{g}_{i j}=g_{i j}+a_{i j}$ of index $k$, with $\widehat{a}=$ $c^{-1} \widehat{g}_{ \pm} \widehat{F}$ and respective nonvanishing on $\mathbf{V}^{n+n}$ scalar function $\nu=\mp c^{2}$ for a given $\left(\widehat{g},{ }_{ \pm} \widehat{F}, \widehat{\xi}, \widehat{\eta}\right)$ structure of index $k$.

Proof. By straightforward computations, we can verify that a sum $g_{i j}+a_{i j}$, where the symmetric and skew-symmetric parts are connected to the $\left(\widehat{g},{ }_{ \pm} \widehat{F}, \widehat{\xi}, \widehat{\eta}\right)$-structure of index $k$ and scalar function in the theorem, is a natural nonsymmetric metric.

The inverse statement also holds true. To show this we note that for modules (2.10) one follows that $\widehat{g} \widehat{m}={ }^{t} \widehat{m} \widehat{g}$ which proves:

Theorem 5.2. For any natural nonsymmetric metric $\check{g}_{i j}=g_{i j}+a_{i j}$ of index $k$ with $\nu=\mp c^{2}$, we can define values the ${ }_{ \pm} \widehat{F}^{2}=\mp c \widehat{g}^{-1} \widehat{a}$ (or, equivalently, ${ }_{ \pm} \widehat{F}^{2}=\mp c^{-1} \check{a} \widehat{g}$ ) which for the quadruplet $\left(g_{i j},{ }_{ \pm} F_{j}^{i}, \xi_{i^{\prime}}^{j}, \eta_{i}^{i^{\prime}}\right)$ states a $\left(\widehat{g},{ }_{ \pm} \widehat{F}, \widehat{\xi}, \widehat{\eta}\right)$-structure of index $k$.

We conclude that the class of natural nonsymmetric metrics naturally generalizes the concepts of (pseudo) Riemannian metrics and of almost Hermitian/hyperbolic structures. 


\subsection{Main theorems for d-connections metric compatibility}

For a fixed d-connection ${ }_{\circ} \mathbf{D}=\left({ }_{\circ} D_{k},{ }_{\circ} D_{c}\right)=\left({ }_{\circ} L_{j k}^{i},{ }_{\circ} C_{b c}^{a}\right)$, we establish the existence and arbitariness of the d-connections which are compatible with a nonsymmetric d-metric $\check{\mathbf{g}}=\mathbf{g}+\mathbf{a}$. Our aim is to redefine the A. Kawaguchi metrization procedure [32, 33, 34] considered in Finsler geometry and developed for Lagrange spaces (including their noncommutative metric generalizations) $[13,14,15]$ for nonholonomic manifolds enabled with nonsymmetric metric structures $[23,16,17]$.

Let us consider a system of tensorial equations for unknown d-tensors ${ }_{h} \mathbf{B}=\left\{B_{r k}^{s}\right\}$ and ${ }_{v} \mathbf{B}=\left\{B_{e c}^{b}\right\}$,

$$
\begin{aligned}
& { }_{h}^{+} O_{h} \mathbf{B}={ }_{h} \mathbf{U}, \quad{ }_{v}^{+} O_{v} \mathbf{B}={ }_{v} \mathbf{U}, \quad{ }_{h}^{+} \Psi_{h} \mathbf{B}={ }_{h} \widetilde{\mathbf{U}}, \quad{ }_{v}^{+} \Psi_{v} \mathbf{B}={ }_{v} \widetilde{\mathbf{U}}, \\
& l_{i}^{r}\left(a_{s j} B_{r k}^{s}+{ }_{\circ} D_{k} a_{r j}\right)=0, \quad l_{a}^{b}\left(a_{b d} B_{e c}^{b}+{ }_{\circ} D_{c} a_{e d}\right)=0, \\
& l_{s}^{i}\left(m_{j}^{r} B_{r k}^{s}+{ }_{\circ} D_{k} l_{j}^{s}\right)=0, \quad l_{b}^{a}\left(m_{d}^{e} B_{e c}^{b}+{ }_{\circ} D_{c} l_{d}^{b}\right)=0,
\end{aligned}
$$

where ${ }_{h} \mathbf{U}=\left\{U_{r k}^{i}\right\},{ }_{v} \mathbf{U}=\left\{U_{e c}^{b}\right\}$ and ${ }_{h} \widetilde{\mathbf{U}}=\left\{\widetilde{U}_{r k}^{s}\right\},{ }_{v} \widetilde{\mathbf{U}}=\left\{\widetilde{U}_{e c}^{b}\right\}$ are given by formulas

$$
2 U_{j k}^{i}=-g^{i r}{ }_{\circ} D_{k} g_{r j}, \quad 2 U_{e c}^{b}=-g^{b d}{ }_{\circ} D_{c} g_{d e},
$$

and

$$
\begin{aligned}
& 2 \widetilde{U}_{j k}^{i}=-\left(\check{a}^{i r}{ }_{\circ} D_{k} a_{r j}+3 l_{s{ }^{\circ}}^{i} D_{k} l_{j}^{s}-{ }_{\circ} D_{k} l_{j}^{i}\right), \\
& 2 \widetilde{U}_{e c}^{b}=-\left(\check{a}^{b d}{ }_{\circ} D_{c} a_{d e}+3 l_{d{ }^{\circ}} D_{c} l_{e}^{d}-{ }_{\circ} D_{c} l_{e}^{b}\right) .
\end{aligned}
$$

One holds:

Theorem 5.3. A d-connection $\mathbf{D}=\left(L_{j k}^{i}, C_{b c}^{a}\right)={ }_{\circ} \mathbf{D}+\mathbf{B}$ is compatible with the nonsymmetric $d$-metric $\check{\mathbf{g}}=\mathbf{g}+\mathbf{a}$ on $\mathbf{V}^{n+n}$ if it is defined by a deformation (distorsion) d-tensor $\mathbf{B}=\left({ }_{h} \mathbf{B},{ }_{v} \mathbf{B}\right)$ which is a solution of d-tensor equations (5.2) for the values $\mathbf{U}=\left({ }_{h} \mathbf{U},{ }_{v} \mathbf{U}\right)$ and $\widetilde{\mathbf{U}}=\left({ }_{h} \widetilde{\mathbf{U}},{ }_{v} \widetilde{\mathbf{U}}\right)$ constructed from the coefficients of the nonsymmetric metric and fixed d-connection ${ }_{\circ} \mathbf{D}$ following, respectively, formulas (5.3) and (5.4).

Proof. We sketch the proof for the h-components (considerations for v-components being similar). The metricity conditions (3.6) are equivalent to

$$
{ }_{\circ} D_{k} g_{i j}+g_{s r} B_{j k}^{r}+g_{r j} B_{s k}^{r}=0 \quad \text { and } \quad{ }_{\circ} D_{k} a_{i j}+a_{s r} B_{j k}^{r}+a_{r j} B_{s k}^{r}=0 .
$$

Contracting the first equation with $g^{s i}$ we get the first equation from (5.2). Contracting the second equation with $\check{a}^{i r}$ and $l_{i}^{r}$ and taking into account the the compatibility of $\mathbf{D}$ with $\check{\mathbf{g}}$ means $l_{s}^{i} D_{k} l_{j}^{s}=0$, one obtains the firsts equations from the third and forth rows in (5.2) and the equation ${ }_{h}^{+} \Psi_{h} \mathbf{B}={ }_{h} \widehat{\mathbf{U}}$, where $2 \widehat{U}_{j k}^{i}=-\left(\check{a}^{i r}{ }_{\circ} D_{k} a_{r j}+l_{s \circ}^{i} D_{k} l_{j}^{s}\right)$. Following Proposition 4.4, the last equation is equivalent to the first equation in the second row in (5.2) with ${ }_{h} \widetilde{\mathbf{U}}={ }_{h} \widehat{\mathbf{U}}+2_{h} \theta_{h} \widehat{\mathbf{U}}$, where the values (5.3) are obtained by re-grouping the coefficients.

One can be proposed a further simplification of such geometric models:

Definition 5.2. A natural nonsymmetric metric of index $k$ is of elliptic (hyperbolic) type if $\nu=-c_{0}^{2}\left(\nu=c_{0}^{2}\right)$, where $c_{0}$ is a nonzero constant.

This definition is suggested by Theorems 4.2, 5.1 and 5.2 and Proposition 3.3:

Corollary 5.1. The function $\nu(x, y)$ in equation (4.11) is a nonzero constant, $\nu(x, y)=\mp c_{0}^{2}$, if the d-connection $\mathbf{D}$ is compatible with the normal nonsymmetric metric $\check{\mathbf{g}}$. 
Proof. One follows from equations (3.6) and Proposition 3.3 that $\left(\mathbf{D}_{\alpha} \nu\right) a_{i j}=0$ which means that $\left(\mathbf{D}_{\alpha} \nu\right)=0$ because $a_{i j} \check{a}^{j i}=n-k \neq 0$. This is possible if $\nu$ is a nonzero constant.

One holds true the inverse statement of Theorem 5.3:

Theorem 5.4. For a natural nonsymmetric metric $\breve{\mathbf{g}}$ of index $k$, the values $\mathbf{U}=\left({ }_{h} \mathbf{U},{ }_{v} \mathbf{U}\right)$ and $\widetilde{\mathbf{U}}=\left({ }_{h} \widetilde{\mathbf{U}},{ }_{v} \widetilde{\mathbf{U}}\right)$ defined respectively by formulas (5.3) and (5.4) satisfy the equations

$$
\begin{array}{ccc}
{ }_{h}^{+} O_{h} \mathbf{U}=0, & { }_{h}^{+} \Psi_{h} \widetilde{\mathbf{U}}=0, & { }_{h} \Psi_{h} \mathbf{U}={ }_{h}^{-} O_{h} \widetilde{\mathbf{U}}, \\
{ }_{v}^{+} O_{v} \mathbf{U}=0, & { }_{v}^{+} \Psi_{v} \widetilde{\mathbf{U}}=0, & { }_{v}^{-} \Psi_{v} \mathbf{U}={ }_{v} O_{v} \widetilde{\mathbf{U}} .
\end{array}
$$

A proof of this Theorem consists from straightforward verifications that $\mathbf{U}$ and $\widetilde{\mathbf{U}}$ really solves the equations (5.5) for given values of Obata operators $\mathbf{O}$ and $\Psi$ constructed respectively from the components of symmetric and skew-symmetric parts of nonsymmetric metric (in [15] it is contained a similar proof for the so-called nonsymmetric Eisenhart-Lagrange metric on tangent bundles).

Example 5.1. By direct computations, we can check that for any given d-connection ${ }_{\circ} \Gamma_{\beta \gamma}^{\alpha}=$ $\left({ }_{\circ} L_{j k}^{i},{ }_{\circ} C_{b c}^{a}\right)$ and nonsymmetric d-metric $\check{\mathbf{g}}=\mathbf{g}+\mathbf{a}$ on $\mathbf{V}^{n+n}$ the d-connection ${ }_{*} \Gamma_{\beta \gamma}^{\alpha}=\left({ }_{*} L_{j k}^{i},{ }_{*} C_{b c}^{a}\right)$, where

$$
\begin{aligned}
& { }_{*} L_{j k}^{i}={ }_{\circ} L_{j k}^{i}+\frac{1}{2}\left[g^{i r}{ }_{\circ} D_{k} g_{r j}+{ }^{ \pm} O_{s j}^{i r}\left(\check{a}^{s t}{ }_{\circ} D_{k} a_{t r}+3 l_{t \circ}^{s} D_{k} l_{r}^{t}-{ }_{\circ} D_{k} l_{r}^{s}\right)\right], \\
& { }_{*} C_{b c}^{a}={ }_{\circ} C_{b c}^{a}+\frac{1}{2}\left[g^{a h}{ }_{\circ} D_{c} g_{h b}+{ }^{ \pm} O_{e b}^{a h}\left(\check{a}^{e d}{ }_{\circ} D_{c} a_{d h}+3 l_{d{ }^{\circ}}^{e} D_{c} l_{h}^{d}-{ }_{\circ} D_{c} l_{h}^{e}\right)\right]
\end{aligned}
$$

is d-metric compatible, i.e. satisfies the conditions ${ }_{*} \mathbf{D} \check{\mathbf{g}}=0$.

In a more general case, one holds:

Theorem 5.5. The set of $d$-connections $\mathbf{D}={ }_{\circ} \mathbf{D}+\mathbf{B}$ being generated by deformations of an arbitrary fixed d-connection ${ }_{\circ} \mathbf{D}$ in order to be compatible with a given nonsymmetric $d$-metric $\check{\mathbf{g}}=\mathbf{g}+\mathbf{a}$ on $\mathbf{V}^{n+n}$ is defined by distorsion d-tensors $\mathbf{B}=\left({ }_{h} \mathbf{B},{ }_{v} \mathbf{B}\right)$ of type

$$
{ }_{h} \mathbf{B}={ }_{h} \mathbf{U}+{ }_{h}^{+} O\left({ }_{h} \widetilde{\mathbf{U}}+{ }_{h}^{+} \Psi \mathbf{Y}\right) \quad \text { and } \quad{ }_{v} \mathbf{B}={ }_{v} \mathbf{U}+{ }_{v}^{+} O\left({ }_{v} \widetilde{\mathbf{U}}+{ }_{v}^{+} \Psi \mathbf{Z}\right),
$$

or

$$
{ }_{h} \mathbf{B}={ }_{h} \widetilde{\mathbf{U}}+{ }_{h}^{+} \Psi\left({ }_{h} \mathbf{U}+{ }_{h}^{+} O \mathbf{Y}\right) \quad \text { and } \quad{ }_{v} \mathbf{B}={ }_{v} \widetilde{\mathbf{U}}+{ }_{v}^{+} \Psi\left({ }_{v} \mathbf{U}+{ }_{v}^{+} O \mathbf{Z}\right),
$$

where $\mathbf{Y}$ and $\mathbf{X}$ are arbitrary d-tensor fields of type $(1,2)$.

Proof. It follows from Theorems 5.3 and 5.4.

For the statements of Example 5.1, we have:

Remark 5.1. The formulas for the metric compatible d-connection ${ }_{*} \boldsymbol{\Gamma}_{\beta \gamma}^{\alpha}(5.6)$ consist a particular case when the deformation d-tensor $\mathbf{B}=\left({ }_{h} \mathbf{B},{ }_{v} \mathbf{B}\right)$ is computed by (5.7), or (5.8), for $\mathbf{Y}=0$ and $\mathbf{Z}=0$.

From the Corollary 5.1 and Theorem 5.5, we get:

Conclusion 5.1. The set of all d-connections $\boldsymbol{\Gamma}_{\beta \gamma}^{\alpha}=\left(L_{j k}^{i}, C_{b c}^{a}\right)$ being compatible to a nonsymmetric metric $\mathbf{g}$ of elliptic/hyperbolic type on $\mathbf{V}^{n+n}$ is parametrized by formulas

$$
L_{j k}^{i}={ }_{*} L_{j k}^{i}+{ }^{+} O_{s j}^{i r+} \Psi_{p r}^{s m} \mathbf{Y}_{m k}^{p} \quad \text { and } \quad C_{b c}^{a}={ }_{*} C_{b c}^{a}+{ }^{+} O_{e b}^{a h+} \Psi_{f h}^{e d} \mathbf{Z}_{d c}^{f},
$$

where ${ }_{*} L_{j k}^{i}$ and ${ }_{*} C_{b c}^{a}$ are given respectively by formulas $(5.6),{ }_{h}^{+} \Psi=\left\{{ }^{+} \Psi_{p r}^{s m}\right\}$ and ${ }_{v}^{+} \Psi=\left\{{ }^{+} \Psi_{f h}^{e d}\right\}$ and $\mathbf{Y}=\left\{Y_{m k}^{p}\right\}$ and $\mathbf{Z}=\left\{Z_{d c}^{f}\right\}$ are correspondingly arbitrary horizontal and vertical d-tensors of type $(1,2)$. 
To chose a parametrization that $\nu$ is constant is the simplest way to prove formulas (5.9) defining the set of all d-connections being compatible to a given nonsymmetric metric.

Finally, we note that because ${ }_{\circ} \Gamma_{\beta \gamma}^{\alpha}=\left({ }_{0} L_{j k}^{i},{ }_{\circ} C_{b c}^{a}\right)$ is an arbitrary d-connection, we can chose it to be an important one for certain physical or geometrical problems. For instance, in Section 7 we shall consider that ${ }_{\circ} \Gamma_{\beta \gamma}^{\alpha}$ is defined by the Cartan d-connection constructed for Lagrange/Finsler spaces for a symmetric d-metric $g$ corresponding to the Lagrange/Finsler d-metric which will allow us to perform certain nonsymmetric generalizations of such geometries. In our further researches, we are going to consider certain exact solutions in gravity with nonholonomic variables defining a corresponding ${ }_{\circ} \Gamma_{\beta \gamma}^{\alpha}$ and then deformed to nonsymmetric configurations following formulas (5.6) and/or (5.9).

\section{$6 \quad$ Nonsymmetric gravity and nonholonomic frames}

A nonsymmetric gravitational theory (NGT) based on decompositions of the nonsymmetric metric $\check{g}_{\mu \nu}$ and affine connection $\Gamma_{\beta \gamma}^{\alpha}$ was elaborated in series of work by J. Moffat and coauthors, see $[5,6,7,8,9,10]$ and references therein ${ }^{4}$.

In this section, we show how NGT can be formulated on $\mathrm{N}$-anholonomic manifolds where an additional geometric structure (the N-connection) is present and the geometric constructions can be equivalently (at least at classical level) performed in $\mathrm{N}$-adapted or not $\mathrm{N}$-adapted forms.

We note that for a class of geometric and physical models the N-connection coefficients are induced by certain subsets of generic off-diagonal coefficients of the metric. In this approach, the $\mathrm{N}$-connection splitting results in a nonholonomic decompositon of geometric objects with respect to certain frames with mixed holonomic-nonholonomic basic vectors which may be convenient for constructing, for instance, new classes of exact solutions, to define spacetimes with generalized symmetries, nonholomogeneity and effective local anisotropy, or in order to elaborate certain models of gauge type and/or geometric/deformation quantization.

In a more general context, we can consider that the $\mathrm{N}$-connection is defined by an additional geometric structure (independent from the metric and linear connection structures), like in generalized Lagrange-Finsler theories, when certain geometric or physical/mechanical theories are defined on nonholonomic manifolds. Nevertheless, even in such cases, following the method of metrization of d-connections considered in the previous section, we can redefine the constructions for nonholonomic (pseudo) Riemann or Riemann-Cartan with additional effective field interactions and nonholonomic constraints. As a matter of principle, we can use the Levi-Civita connection working with non $\mathrm{N}$-adapted geometric objects.

\subsection{On (not) N-adapted models of NGT}

Let us consider a d-connection $\boldsymbol{\Gamma}_{\beta \gamma}^{\alpha}$ not obligatory compatible to a nonsymmetric metric $\check{\mathbf{g}}=$ $\left\{\check{\mathbf{g}}_{\alpha \beta}\right\}$ (2.7) on $\mathbf{V}^{2+2}$ enabled with an arbitrary N-connection structure $\mathbf{N}=\left\{N_{i}^{a}\right\}(2.2)^{5}$. We can introduce nonsymmetric gravitational equations following the approach elaborated in [6], but in our case working with respect to N-adapted bases (2.3) and (2.4).

\footnotetext{
${ }^{4}$ we note that in this paper we use a different system of denotations; the final version of NGT proposed by J. Moffat's group is free of ghosts, tachions and higher-order poles in the propagator in the linear approximation on Minkowski space; an expansion of the general nonsymmetric metric about an arbitrary Einstein background metric yields field equations to first order in the skew-symmetric part of metric, which are free of coupling to unphysical (negative energy) modes; the solutions of such gravitational field equations have consistent asymptotic boundary conditions; here, it should be noted that in the mentioned works a set of theoretical and experimental data were explained and in consistent way by NGT and its further modifications.

${ }^{5}$ For our purposes, we consider four dimensional spacetimes with $2+2$ nonholonomic splitting.
} 
For any system of reference, we can write

$$
g_{\mu \nu}=\check{g}_{(\mu \nu)}=\frac{1}{2}\left(\check{g}_{\mu \nu}+\check{g}_{\mu \nu}\right), \quad a_{\mu \nu}=\check{g}_{[\mu \nu]}=\frac{1}{2}\left(\check{g}_{\mu \nu}-\check{g}_{\mu \nu}\right), \quad \Gamma_{\mu \nu}^{\lambda}=\Gamma_{(\mu \nu)}^{\lambda}+\Gamma_{[\mu \nu]}^{\lambda} .
$$

Introducing the unconstrained (nonsymmetric) affine connection

$$
W_{\mu \nu}^{\lambda} \doteqdot \Gamma_{\mu \nu}^{\lambda}-\frac{2}{3} \delta_{\mu}^{\lambda} W_{\nu}
$$

where $W_{\nu}=\frac{1}{2}\left(W_{\mu \lambda}^{\lambda}-W_{\lambda \mu}^{\lambda}\right)$, which leads to the condition $\Gamma_{\mu}=\Gamma_{[\mu \lambda]}^{\lambda}=0$. We note that the coefficients of $\Gamma_{\mu \nu}^{\lambda}$ and $W_{\mu \nu}^{\lambda}$ are given with respect to N-adapted bases (2.3) and (2.4) but we do not use for such values "boldfaced" symbols because we have not supposed that these linear connections are adapted to the N-connection splitting (2.1).

The contracted curvature tensors for the above linear connections are related by formulas

$$
{ }_{W} R_{\mu \nu}={ }_{\Gamma} R_{\mu \nu}+\frac{2}{3} \mathbf{e}_{[\nu} W_{\mu]},
$$

where

$$
{ }_{\Gamma} R_{\mu \nu}=\mathbf{e}_{\beta} \Gamma_{\mu \nu}^{\beta}-\frac{1}{2}\left(\mathbf{e}_{\nu} \Gamma_{(\mu \lambda)}^{\lambda}+\mathbf{e}_{\mu} \Gamma_{(\nu \lambda)}^{\lambda}\right)-\Gamma_{\alpha \nu}^{\beta} \Gamma_{\mu \beta}^{\alpha}+\Gamma_{(\alpha \lambda)}^{\lambda} \Gamma_{\mu \nu}^{\alpha} .
$$

The field equations of N-anholonomic NGT in presence of a source $\Upsilon_{\mu \nu}$ for matter fields are

$$
\begin{aligned}
& { }_{W} G_{\mu \nu}+\lambda \check{\mathbf{g}}_{\mu \nu}+\frac{\mu^{2}}{4} \mathbf{S}_{\mu \nu}-\frac{1}{6}{ }_{P} G_{\mu \nu}=8 \pi \Upsilon_{\mu \nu}, \\
& 2 \mathbf{e}_{\nu}\left(\sqrt{|\check{\mathbf{g}}|} \check{\mathbf{g}}^{[\nu \mu]}\right)=\sqrt{|\check{\mathbf{g}}|} \check{\mathbf{g}}^{(\nu \mu)} W_{\nu}, \\
& (\sqrt{|\check{\mathbf{g}}|})^{-1} \mathbf{e}_{\sigma}\left(\sqrt{|\check{\mathbf{g}}|} \check{\mathbf{g}}^{\mu \nu}\right)+\check{\mathbf{g}}^{\rho \nu} W_{\rho \sigma}^{\mu}-\check{\mathbf{g}}^{\mu \nu} W_{\rho \sigma}^{\rho}+\frac{W_{\beta}}{6}\left(\delta_{\sigma}^{\nu} \check{\mathbf{g}}^{(\mu \beta)}+\delta_{\sigma}^{\mu} \check{\mathbf{g}}^{(\nu \beta)}\right)+\frac{2}{3} \delta_{\sigma}^{\nu} \check{\mathbf{g}}^{\mu \rho} W_{[\rho \beta]}^{\beta}=0,
\end{aligned}
$$

where $|\check{\mathbf{g}}| \doteqdot \operatorname{det}\left|\check{\mathbf{g}}_{\mu \nu}\right|, \check{\mathbf{g}}^{\mu \nu} \check{\mathbf{g}}_{\sigma \nu}=\check{\mathbf{g}}^{\nu \mu} \check{\mathbf{g}}_{\nu \sigma}=\delta_{\sigma}^{\mu}$ (we use boldface indices for the nonsymmetric metric and bases (2.3) and (2.4) because they can be adapted to the $\mathrm{N}$-connection structure even a general linear connection and related tensors are not distinguished); $\lambda$ is the cosmological constant and $\mu^{2}$ is an additional cosmological constant associated to to $\mathbf{a}_{\mu \nu}$ (there are used the physical units when the gravitational and vacuum speed are stated to be dimensionless and equal to unity);

$$
\begin{aligned}
& \mathbf{S}_{\mu \nu} \doteqdot \mathbf{a}_{\mu \nu}+\check{\mathbf{g}}^{[\rho \sigma]}\left(\check{\mathbf{g}}_{\mu \sigma} \check{\mathbf{g}}_{\rho \nu}+\frac{1}{2} \mathbf{a}_{\sigma \rho} \check{\mathbf{g}}_{\mu \nu}\right) \\
& { }_{W} G_{\mu \nu} \doteqdot{ }_{W} R_{\mu \nu}-\frac{1}{2} \check{\mathbf{g}}_{\mu \nu W} \overleftarrow{R}, \quad{ }_{P} G_{\mu \nu} \doteqdot P_{\mu \nu}-\frac{1}{2} \check{\mathbf{g}}_{\mu \nu} P
\end{aligned}
$$

for $P_{\mu \nu} \doteqdot W_{\mu} W_{\nu}$ and $P \doteqdot \check{\mathbf{g}}^{\mu \nu} P_{\mu \nu}=\check{\mathrm{g}}^{(\mu \nu)} P_{\mu \nu}$, when the scalar curvatures are defined respectively $_{W} \overleftarrow{R} \doteqdot \check{\mathbf{g}}^{\mu \nu}{ }_{W} R_{\mu \nu},{ }_{\Gamma} \overleftarrow{R} \doteqdot \check{\mathbf{g}}^{\mu \nu}{ }_{\Gamma} R_{\mu \nu}, \ldots$ We note that, in general, the h- and v-components of $\check{\mathbf{g}}^{[\rho \sigma]}$ are different from $\widetilde{a}^{-1}(2.13)$ because we have not yet introduced here metric d-connections and complete $\mathrm{N}$-adapted and d-tensor calculus.

The matter fields d-tensor $\Upsilon_{\mu \nu}$ from the first equation in (6.2) is constrained to satisfy the so-called matter response equations,

$$
\check{\mathbf{g}}_{\mu \rho} \mathbf{e}_{\nu}\left(\sqrt{|\check{\mathbf{g}}|} \Upsilon^{\mu \nu}\right)+\check{\mathbf{g}}_{\rho \mu} \mathbf{e}_{\nu}\left(\sqrt{|\check{\mathbf{g}}|} \Upsilon^{\nu \mu}\right)+\left(\mathbf{e}_{\nu} \check{\mathbf{g}}_{\mu \rho}+\mathbf{e}_{\mu} \check{\mathbf{g}}_{\rho \nu}-\mathbf{e}_{\rho} \check{\mathbf{g}}_{\mu \nu}\right) \sqrt{|\check{\mathbf{g}}|} \Upsilon^{\nu \mu}=0
$$

which is a consequence of the generalized Bianchi identities

$$
\mathbf{e}_{\mu}\left[\sqrt{|\check{\mathbf{g}}|} \check{\mathbf{g}}^{\mu \nu}{ }_{\Gamma} G_{\rho v}+\sqrt{|\check{\mathbf{g}}|} \check{\mathbf{g}}^{\nu \mu}{ }_{\Gamma} G_{v \rho}\right]+\sqrt{|\check{\mathbf{g}}|} G_{\mu v} \mathbf{e}_{\rho} \check{\mathbf{g}}^{\mu \nu}=0,
$$


see a detailed study in [35, 6], where a variational proof of equations (6.2) and (6.4) is formulated by fixing a corresponding coefficient before $P_{\mu \nu}$ in order to yield a consistent theory with ghost and tachyon free perturbative solutions to the field equations.

It should be emphasized that different models of nonsymmteric theory of gravity were elaborated in such a way that the induced general linear connection is not metric compatible. In our approach, we shall prove that it is possible to elaborate the nonsymmetric gravity theory in a general metric compatible form by using corresponding classes of d-connections. This is very important from physical point of view (there is not a well accepted interpretation of nonmetricity fields) and presents certain interests from the viewpoint of the Ricci flow theory with nonholonomic constraints $[17,23]$ when in the simplest approach a symmetric metric can evolve into a nonsymetric one, and inversely, but preserving the general metric compatibility of linear connection which is very important for definition of conservation laws and related physical values.

Theorem 6.1. We obtain a canonical d-metric compatible and $N$-adapted nonholonomic NGT completely defined by a $N$-connection $\mathbf{N}=\left\{N_{i}^{a}\right\}$ and d-metric $\check{\mathbf{g}}=\mathbf{g}+\mathbf{a}$ (2.7) of elliptic/hyperbolic type if we chose instead of arbitrary affine connection $\Gamma_{\mu \nu}^{\lambda}$ the metric compatible $d$-connection ${ }_{*} \Gamma_{\beta \gamma}^{\alpha}=\left({ }_{*} L_{j k}^{i},{ }_{*} C_{b c}^{a}\right)(5.6)$.

Proof. We sketch the idea of the proof which can be obtained by a N-adapted variational calculus, similar to that from [35] when instead of partial derivatives there are used the "Nelongated" partial derivatives $\mathbf{e}_{\rho}(2.3)$, varying independently the d-fields $\check{\mathbf{g}}=\mathbf{g}+\mathbf{a}$ and ${ }_{*} \boldsymbol{\Gamma}_{\beta \gamma}^{\alpha}$. In this case, $\check{a}=\left(\check{a}^{i j}\right)$ does not depend on the choice of fields $\widehat{\xi}$, see (2.13), and we can write $\check{\mathbf{g}}^{[\rho \sigma]}=\check{\mathbf{a}}^{\rho \sigma}=\left[\check{a}^{i j}, \check{a}^{c b}\right]$, where $\check{a}^{i j}=-\check{a}^{j i}$ and $\check{a}^{c b}=-\check{a}^{b c}$. Instead of an affine connection (6.1) we work with metric d-connections,

$$
{ }_{*} \mathbf{W}_{\mu \nu}^{\lambda} \doteqdot{ }_{*} \boldsymbol{\Gamma}_{\mu \nu}^{\lambda}-\frac{2}{3} \delta_{\mu *}^{\lambda} \mathbf{W}_{\nu}
$$

where ${ }_{*} \mathbf{W}_{\nu}=\frac{1}{2}\left({ }_{*} \mathbf{W}_{\mu \lambda}^{\lambda}-{ }_{*} \mathbf{W}_{\lambda \mu}^{\lambda}\right)$, and we use boldface symbols. The corresponding Ricci dtensors are related by formulas

$$
{ }_{W}^{*} \mathbf{R}_{\mu \nu}={ }_{\Gamma}^{*} \mathbf{R}_{\mu \nu}+\frac{2}{3} \mathbf{e}_{[\nu}^{*} \mathbf{W}_{\mu]},
$$

where

$$
{ }_{\Gamma}^{*} \mathbf{R}_{\mu \nu}=\mathbf{e}_{\beta *} \Gamma_{\mu \nu}^{\beta}-\frac{1}{2}\left(\mathbf{e}_{\nu *} \Gamma_{(\mu \lambda)}^{\lambda}+\mathbf{e}_{\mu *} \Gamma_{(\nu \lambda)}^{\lambda}\right)-{ }_{*} \Gamma_{\alpha \nu *}^{\beta} \Gamma_{\mu \beta}^{\alpha}+{ }_{*} \Gamma_{(\alpha \lambda) *}^{\lambda} \Gamma_{\mu \nu}^{\alpha} .
$$

has h- and v-components of type (A.3).

The canonical N-adapted field equations for the nonholonomic NGT are

$$
\begin{aligned}
& { }_{W}^{*} \mathbf{G}_{\mu \nu}+\lambda \check{\mathbf{g}}_{\mu \nu}+\frac{\mu^{2}}{4}{ }_{*} \mathbf{S}_{\mu \nu}-\frac{1}{6}{ }_{P} \mathbf{G}_{\mu \nu}=8 \pi \mathbf{\Upsilon}_{\mu \nu}, \\
& 2 \mathbf{e}_{\nu}\left(\sqrt{|\check{\mathbf{g}}|} \check{\mathbf{a}}^{[\nu \mu]}\right)=\sqrt{|\check{\mathbf{g}}|} \check{\mathbf{g}}^{(\nu \mu)}{ }_{*} \mathbf{W}_{\nu}, \\
& (\sqrt{|\check{\mathbf{g}}|})^{-1} \mathbf{e}_{\sigma}\left(\sqrt{|\check{\mathbf{g}}|} \check{\mathbf{g}}^{\mu \nu}\right)+\check{\mathbf{g}}^{\rho \nu}{ }_{*} \mathbf{W}_{\rho \sigma}^{\mu}-\check{\mathbf{g}}^{\mu \nu}{ }_{*} \mathbf{W}_{\rho \sigma}^{\rho} \\
& \quad+\frac{{ }^{*} \mathbf{W}_{\beta}}{6}\left(\delta_{\sigma}^{\nu} \check{\mathbf{g}}^{(\mu \beta)}+\delta_{\sigma}^{\mu} \check{\mathbf{g}}^{(\nu \beta)}\right)+\frac{2}{3} \delta_{\sigma}^{\nu} \check{\mathbf{g}}^{\mu \rho}{ }_{*} \mathbf{W}_{[\rho \beta]}^{\beta}=0,
\end{aligned}
$$

where the formulas for geometric objects and conservation laws are defined, respectively, similarly to (6.3) and (6.4) but for boldfaced d-connections (6.5). These equations can be derived 
from the Lagrangian density

$$
\begin{aligned}
& { }_{*} \mathcal{L}_{\mathrm{NGT}}={ }_{*} \mathcal{L}_{R}+\mathcal{L}_{M}, \\
& { }_{*} \mathcal{L}_{R}=\sqrt{|\check{\mathbf{g}}|} \check{\mathbf{g}}^{\rho \nu *}{ }_{W} \mathbf{R}_{\mu \nu}-2 \lambda \sqrt{|\check{\mathbf{g}}|}-\frac{\mu^{2}}{4} \sqrt{|\check{\mathbf{g}}|} \check{\mathbf{g}}^{\mu \nu} \mathbf{a}_{\mu \nu}-\frac{1}{6} \check{\mathbf{g}}^{\mu \nu}{ }_{*} \mathbf{W}_{\mu *} \mathbf{W}_{\nu}, \\
& \mathcal{L}_{M}=-8 \pi \check{\mathbf{g}}^{\mu \nu} \boldsymbol{\Upsilon}_{\mu \nu}
\end{aligned}
$$

following a $\mathrm{N}$-adapted variational calculus.

The above considerations motivate the concepts:

Definition 6.1. A Moffat gravity (spacetime) model is defined by a nonsymmetric metric $\check{\mathbf{g}}=\left\{\check{\mathbf{g}}_{\alpha \beta}\right\}$ (2.7) and an affine connection $W_{\mu \nu}^{\lambda} \doteqdot \Gamma_{\mu \nu}^{\lambda}-\frac{2}{3} \delta_{\mu}^{\lambda} W_{\nu}$ (6.1) solving the NGT field equations (6.2).

In a more general case, we have

Definition 6.2. A canonical nonholonomic Eisenhart-Moffat gravity (spacetime) model is defined by a fixed N-connection structure $\mathbf{N}$ and a nonsymmetric metric $\check{\mathbf{g}}^{2}=\left\{\check{\mathbf{g}}_{\alpha \beta}\right\}$ (2.7) and a metric compatible d-connection ${ }_{*} \Gamma_{\beta \gamma}^{\alpha}=\left({ }_{*} L_{j k}^{i},{ }_{*} C_{b c}^{a}\right)$ (5.6) solving the N-adapted NGT field equations (6.6).

As a matter of principle, following the Kawaguchi's metrization method we can work with various classes of metric or nonmetric d-connections:

Remark 6.1. Analogous of Theorem 6.1 can be formulated and proven for any metric noncompatible d-connection ${ }_{\circ} \mathbf{D}=\left({ }_{\circ} D_{k},{ }_{\circ} D_{c}\right)=\left({ }_{\circ} L_{j k}^{i},{ }_{\circ} C_{b c}^{a}\right)$, or for any metric compatible d-connection $\boldsymbol{\Gamma}_{\beta \gamma}^{\alpha}=\left(L_{j k}^{i}, C_{b c}^{a}\right)(5.9)$. In all cases, we get N-adapted models of NGT but for ${ }_{\circ} \mathbf{D}$ we generate geometric constructions for nonmetric spaces and for $\boldsymbol{\Gamma}_{\beta \gamma}^{\alpha}$ the models depend on d-tensor fields of type $(1,2)$ for which one has to provide additional geometric and physical motivations.

Here we emphasize that it is preferred to work with metric compatible connection for global definition of spinors and noncommutative versions with Dirac operators in different models of gravity because metric compatibility results in compatible structure between spinor connections and generating Clifford structures bases.

From the last Theorem and Remark, one follows:

Corollary 6.1. If the $N$-connection structure is induced, for instance, by the off-diagonal coefficients of the symmetric part of the nonsymmetric metric, and the canonical nonholonomic Eisenhart-Moffat gravity (which is a metric compatible theory) is equivalent to the Moffat's gravity theory (which was performed in a metric noncompatible form).

Proof. We state that the symmetric part $\mathbf{g}=\mathbf{g}_{\alpha \beta} \mathbf{e}^{\alpha} \otimes \mathbf{e}^{\beta}=g_{i j} e^{i} \otimes e^{j}+g_{a b} \mathbf{e}^{a} \otimes \mathbf{e}^{b}$ in (2.7) with respect to a coordinate base $e^{\alpha}=d u^{\alpha}=\left(d x^{i}, d y^{a}\right)$, is given in the form

$$
\mathbf{g}=\underline{g}_{\alpha \beta}(u) d u^{\alpha} \otimes d u^{\beta}
$$

where

$$
\underline{g}_{\alpha \beta}=\left[\begin{array}{cc}
g_{i j}+N_{i}^{a} N_{j}^{b} h_{a b} & N_{j}^{e} h_{a e} \\
N_{i}^{e} h_{b e} & h_{a b}
\end{array}\right] .
$$

induces the coefficients of $\mathbf{N}$-connection $\mathbf{N}=N_{i}^{a}(u) d x^{i} \otimes \partial / \partial y^{a}(2.2)$. The next step, is to take ${ }_{\circ} \Gamma_{\beta \gamma}^{\alpha}={ }_{n} \boldsymbol{\Gamma}_{\beta \gamma}^{\alpha}$, see Definition 3.2, in ${ }_{*} \Gamma_{\beta \gamma}^{\alpha}{ }^{6}{ }^{6}$ Using the deformation of connection ${ }_{n} \boldsymbol{\Gamma}_{\beta \gamma}^{\alpha}=$

\footnotetext{
${ }^{6}$ In a more general, or special, approach, we can use the d-connections (4.2), or (3.4).
} 
$\Gamma_{\beta \gamma}^{\alpha}+S_{\beta \gamma}^{\alpha}$, we can redefine the N-adapted field equations (6.6) in terms of connection $\Gamma_{\beta \gamma}^{\alpha}$ and $S_{\beta \gamma}^{\alpha}$. The last term can be encoded in terms of d-tensor fields of type $(1,2)$. This way a model of metric compatible and N-adapted NGT is transformed in a Moffat type model of gravity.

The NGT was proven to generate physically consistent models using linear approximations for the nonsymmetric metric about Minkowski and Einstein spaces, see [35, 6] and references therein. Various types of approximations can be performed following a corresponding N-adapted calculus with respect to a nonholonomic background. Depending on the type of background and constraints on nonsymmetric metric components, we obtain different effective models of scalar/vector/tensor gravity, with variable/running physical constants which are intensively exploited in modern cosmology, for instance, see $[10,11]$. To work with nonholonomic backgrounds presents a substantial interest both from conceptual and technical point of views: we can 'extract' from NGT new classes of nonholonomic Einstein, generalized Finsler-Lagrange, ... spaces [24], establish certain new links and develop new methods in geometric quantization [31] and noncommutative gravity $[9,29,30]$, as well to elaborate new methods of constructing exact solutions in Einstein and generalized gravity theories and Ricci flows [24, 18, 19, 20, 21, 22].

\subsection{Expansion of field equations in NGT with respect to nonholonomic backgrounds}

Let us consider the expansion of the field equations (6.6) for $\check{\mathrm{g}}=\mathbf{g}+\mathbf{a}$ around a background spacetime defined by a symmetric d-connection $\mathbf{g}=\left\{\mathbf{g}_{\alpha \beta}\right\}$ and a metric compatible d-connection $\widehat{\boldsymbol{\Gamma}}_{\beta \gamma}^{\alpha}$ defined by $\mathbf{N}$ and $\mathbf{g}$ (it can be a normal, the canonical d-connection, the Cartan or another one) and denote $\check{\mathbf{g}}_{[\alpha \beta]}=\mathbf{a}_{\alpha \beta} \cdot{ }^{7}$ We shall compute

$$
\begin{aligned}
& \check{\mathbf{g}}_{\alpha \beta}=\mathbf{g}_{\alpha \beta}+{ }^{1} \mathbf{g}_{\alpha \beta}+\cdots, \quad \mathbf{a}_{\alpha \beta}={ }^{1} \mathbf{a}_{\alpha \beta}+{ }^{2} \mathbf{a}_{\alpha \beta}+\cdots, \\
& \boldsymbol{\Gamma}_{\beta \gamma}^{\alpha}=\widehat{\boldsymbol{\Gamma}}_{\beta \gamma}^{\alpha}+{ }^{1} \boldsymbol{\Gamma}_{\beta \gamma}^{\alpha}+\cdots,
\end{aligned}
$$

Substituting into field equations, for $\lambda=0$ and $\boldsymbol{\Upsilon}_{\mu \nu}=0$, we get to first order on fields, with respect to $\mathrm{N}$-adapted bases (2.3) and (2.4),

$$
\begin{aligned}
& \widehat{\mathbf{R}}_{\alpha \beta}=0, \\
& 2 \widehat{\mathbf{D}}^{\nu} \mathbf{a}_{\mu \nu}=-\mathbf{W}_{\mu}, \\
& \left(\widehat{\square}+\mu^{2}\right) \mathbf{a}_{\mu \nu}=2 \widehat{\mathbf{R}}_{\cdot \nu \cdot \mu}^{\sigma \cdot \beta} \mathbf{a}_{\beta \sigma}+\frac{1}{3} \widehat{\mathbf{D}}_{[\mu} \mathbf{W}_{\nu]},
\end{aligned}
$$

where $\mathbf{a}_{\alpha \beta}={ }^{1} \mathbf{a}_{\alpha \beta}, \mathbf{W}_{\mu}={ }^{1} \mathbf{W}_{\mu}, \widehat{\square}=\widehat{\mathbf{D}}^{\nu} \widehat{\mathbf{D}}_{\nu}$ for $\widehat{\mathbf{D}}^{\nu}=\mathbf{g}^{\nu \mu} \widehat{\mathbf{D}}_{\mu}$, for $\mathbf{g}^{\nu \mu}$ being inverse to $\mathbf{g}_{\alpha \beta}$ (these d-tensors are used for rasing and lowering indices), and $\widehat{\mathbf{R}}_{\cdot \nu \cdot \mu}^{\sigma \cdot \beta}$ and $\widehat{\mathbf{R}}_{\alpha \beta}$ are respectively curvature and Ricci d-tensors, with h- and v-decompositions, defined by formulas (A.2) and (A.3). Following a d-tensor calculus similar to that in $[35,6]$, but for a canonical background

\footnotetext{
${ }^{7}$ In order to elaborate a consistent "perturbation" theory on fields $\mathbf{a}_{\alpha \beta}$, we may consider that such nonsymmetric deformations of metrics are defined by an additional, or the same Newton constant (but under nonholonomic Ricci flows) used as a small parameter. Such decompositions are used for definition of "week" gravitational waves in general relativity and in "perturbative" models of quasi-classical gravity; in a more general approach, for nonsymmmetric metrics, but in not $\mathrm{N}$-adapted forms, such constructions were introduced and developed in a series of works by J. Moffat and co-authors [5, 6, 7, 8].
} 
d-connection $\widehat{\boldsymbol{\Gamma}}_{\beta \gamma}^{\alpha}$, we can represent (6.7) in the form

$$
\begin{aligned}
& \widehat{\mathbf{R}}_{\alpha \beta}=0, \\
& \widehat{\mathbf{D}}^{\sigma} \mathbf{a}_{\mu \sigma}=\frac{1}{\mu^{2}} \widehat{\mathbf{D}}^{\nu}\left(2 \widehat{\mathbf{R}}_{\cdot \nu \cdot \mu}^{\sigma \cdot \beta} \mathbf{a}_{\sigma \beta}-(\widehat{\mathbf{R}} \mathbf{a})_{\mu \nu}\right), \\
& \left(\widehat{\square}+\mu^{2}\right) \mathbf{a}_{\mu \nu}=\mathbf{M}_{\mu \nu},
\end{aligned}
$$

where

$$
\mathbf{M}_{\mu \nu}=2 \widehat{\mathbf{R}}_{\cdot \nu \cdot \mu}^{\sigma \cdot \beta} \mathbf{a}_{\beta \sigma}+\frac{2}{\mu^{2}} \widehat{\mathbf{D}}_{[\nu} \widehat{\mathbf{D}}^{\rho}\left(2 \widehat{\mathbf{R}}_{\cdot \rho \cdot \mu}^{\sigma \cdot \beta} \mathbf{a}_{\beta \sigma}-(\widehat{\mathbf{R}} \mathbf{a})_{\mu] \rho}\right)
$$

and $(\widehat{\mathbf{B}} \mathbf{a})_{\mu \nu}$ denotes additional terms involving products of the Riemann d-tensor and skewsymmetric part of d-metric.

There were constructed a number of solutions with $\widehat{\mathbf{R}}_{\alpha \beta}=0$, see a review of results in [24], where it is emphasized that we can constrain additionally the integral varieties of these equations in order to generate off-diagonal vacuum solutions in general relativity. They can be used for generalizations in NGT when the background is nonholonomic and/or to model a Finsler/Lagrange like configurations. We constructed such spacetime models with running physical constants and nonsymmetric metrics in [23], where nontrivial values for $\mathbf{a}_{\beta \sigma}$ where defined from the nonholonomic Ricci flow evolution equations. Certain classes of those solutions, when the flow parameter is not identified with a time like coordinate can be constrained additionally to define solutions of (6.8). For instance, the solitonic and pp-wave solutions with vanishing curvature $2 \widehat{\mathbf{R}}_{\cdot \nu \cdot \mu}^{\sigma \cdot \beta}$ at asymptotics result positively in nontrivial solutions for $\mathbf{a}_{\mu \sigma}$ which closely approximate solutions of Proca equations labelled additionally by a Ricci flow parameter.

We conclude that we can perform nonholonomic deformation of symmetric metrics into nonsymmetric ones following the method of anholonomic frames and nonholonomic Ricci flows. Such geometric configurations are also admissible from the viewpoint of metric compatible/noncompatible NGT which presents strong theoretical arguments for physical models with nonsymmetric metrics and nonholonomic configurations.

\section{Gravity and nonsymmetric Lagrange-Finsler spaces}

The approach to geometrization of mechanics on tangent bundles of the R. Miron's school on generalized Lagrange, Hamilton and Finsler geometry is strongly related to the geometry of nonlinear connections on (co) vector/tangent bundles and their higher order generalizations [15, $25,36]$ (this direction was developed as a generalization of the geometry of Finsler and Cartan spaces). From formal point of view any regular mechanics models can modelled as a RiemannCartan geometry with nonholonomic distributions and, inversely, under well defined conditions, gravitational interactions admit an equivalent modelling by (semy) spary configurations for an effective mechanics or nonlinear optics [24]. Here, we note that our approach is different from the so-called analgous gravity [37] where gravitational (for instance, black hole effects) are effectively modelled by heuristic media and flows, but not following a rigorous geometric formalism, for instance, that of N-connections and geometric mechanics.

The aim of this section is to prove that Lagrange-Finsler geometry and nonholonomic gravity can be naturally related to NSG and define certain models of nonsymmetric Lagrange-Finsler geometry. 
Let us consider a regular Lagrangian $L(x, y)=L\left(x^{i}, y^{a}\right)$ modelled on $\mathbf{V}$, when the Lagrange metric (equivalently, Hessian)

$$
{ }^{L} g_{i j}=\frac{1}{2} \frac{\partial^{2} L}{\partial y^{i} \partial y^{j}}
$$

is not degenerated, i.e. $\operatorname{det}\left|g_{i j}\right| \neq 0$,

$\mathrm{N}$-connections were first introduced in Finsler and Lagrange geometry by considering (semi) spray configurations

$$
\frac{d y^{a}}{d \varsigma}+2 G^{a}(x, y)=0
$$

of a curve $x^{i}(\varsigma)$ with parameter $0 \leq \varsigma \leq \varsigma_{0}$, when $y^{i}=d x^{i} / d \varsigma$ [spray configurations are obtained for integrable equations]. One holds the fundamental result (proof is a straightforward computation):

Theorem 7.1. For $4 G^{j}={ }^{L} g^{i j}\left(\frac{\partial^{2} L}{\partial y^{i} \partial x^{k}} y^{k}-\frac{\partial L}{\partial x^{i}}\right)$, with ${ }^{L} g^{i j}$ inverse to ${ }^{L} g_{i j}$, the "nonlinear" geodesic equations (7.2) are equivalent to the Euler-Lagrange equations $\frac{d}{d \varsigma}\left(\frac{\partial L}{\partial y^{i}}\right)-\frac{\partial L}{\partial x^{i}}=0$.

Finsler configurations can be obtained in a particular case when $L(x, y)=F^{2}(x, y)$ for a homogeneous fundamental function $F(x, \lambda y)=\lambda F(x, y), \lambda \in \mathbb{R}$. Lagrange and Finsler geometries can be also modelled on $\mathrm{N}$-anholonomic manifolds [38, 24] provided, for instance, with canonical N-connection structure

$$
{ }^{L} N_{i}^{a}=\frac{\partial G^{a}}{\partial y^{i}} .
$$

Proposition 7.1. A N-connection defines a set of nonholonomic preferred frames

$$
{ }^{L} \mathbf{e}_{\alpha}=\left[{ }^{L} \mathbf{e}_{i}=\frac{\partial}{\partial x^{i}}-{ }^{L} N_{i}^{a}(u) \frac{\partial}{\partial y^{a}}, e_{b}=\frac{\partial}{\partial y^{b}}\right]
$$

and coframes

$$
{ }^{L} \mathbf{e}^{\alpha}=\left[e^{i}=d x^{i}, \mathbf{e}^{a}=d y^{a}+{ }^{L} N_{i}^{a}(x, y) d x^{i}\right] .
$$

Proof. One computes the nontrivial nonholonomy coefficients ${ }^{L} w_{i b}^{a}=\partial^{L} N_{i}^{a} / \partial y^{b}$ and ${ }^{L} w_{i j}^{a}=$ ${ }^{L} \Omega_{j i}^{a}={ }^{L} \mathbf{e}_{i}{ }^{L} N_{j}^{a}-{ }^{L} \mathbf{e}_{j}{ }^{L} N_{i}^{a}$ (where ${ }^{L} \Omega_{j i}^{a}$ are the coefficients of the N-connection curvature) for

$$
\left[{ }^{L} \mathbf{e}_{\alpha},{ }^{L} \mathbf{e}_{\beta}\right]={ }^{L} \mathbf{e}_{\alpha}{ }^{L} \mathbf{e}_{\beta}-{ }^{L} \mathbf{e}_{\beta}{ }^{L} \mathbf{e}_{\alpha}={ }^{L} w_{\alpha \beta}^{\gamma}{ }^{L} \mathbf{e}_{\gamma} .
$$

One holds:

Theorem 7.2. Any regular Lagrange mechanics $L(x, y)=L\left(x^{i}, y^{a}\right)$ can be modelled by the geometry of a $N$-anholonomic manifold $\mathbf{V}^{n+n}$ enabled with $N$-connection ${ }^{L} \mathbf{N}$ and canonical metric structure

$$
{ }^{L} \mathbf{g}={ }^{L} g_{i j}(x, y)\left[e^{i} \otimes e^{j}+{ }^{L} \mathbf{e}^{i} \otimes{ }^{L} \mathbf{e}^{j}\right] .
$$

Proof. For $\mathbf{V}=T M$, the metric (7.4) is just the Sasaki lift of (7.1) on total space; see, for instance, [25]. In abstract form, such canonical constructions can be performed similarly for any $\mathrm{N}$-anholonomic manifold $\mathbf{V}$. This approach to geometric mechanics follows from the fact that the (semi) spray configurations are related to the N-connection structure and defined both by the Lagrangian fundamental function and the Euler-Lagrange equations, see Theorem 7.1. 
It is important:

Proposition 7.2. Any regular Lagrangian $L$ defines on $\mathbf{V}^{n+n}$ a preferred metric compatible $d$-connection structures and metric compatible Lagrange (Finsler, for $L=F^{2}$ ) canonical $d$ connection ${ }^{L} \widehat{\mathbf{D}}$, or normal d-connection ${ }_{n}^{L} \mathbf{D}$, Cartan d-connection ${ }_{c}^{L} \mathbf{D}$.

Proof. We can compute the coefficients of these d-connections by introducing formulas (7.4) and (7.3), respectively, in (4.2), (3.4) and (3.3).

Because, in general, a regular Lagrange mechanics induces a nontrivial N-connection structure, we can consider:

Claim 7.1. There are nonholonomic Ricci flows of Lagrange (Finsler) spaces resulting in additional nonsymmetric components of d-metric (7.4).

Proof. It follows from equation (29) in [23] $\frac{\partial}{\partial \chi}\left(N_{j}^{e} \underline{a}_{b e}\right)$, where $\chi$ is the Ricci flow parameter. Ricci flows of Lagrangians $L(\chi)$ induce flows of ${ }^{L} N_{j}^{e}(\chi)$, which results in nontrivial values of $a_{b e}$. The d-metric (7.4) has to be extended to a variant of d-metric (2.7), when ${ }^{L} \check{\mathbf{g}}={ }^{L} \mathbf{g}+\mathbf{a}$.

The above Claim is supported by

Example 7.1. A class of simplest examples of nonsymmetric Lagrange geometry is generated by a regular Lagrangian $L(x, y)$ and any skew symmetric field $\mathbf{a}$ when ${ }^{L} \check{\mathbf{g}}={ }^{L} \mathbf{g}+\mathbf{a}$ and the d-connection ${ }_{\circ} \Gamma_{\beta \gamma}^{\alpha}=\left({ }_{\circ} L_{j k}^{i},{ }_{\circ} C_{b c}^{a}\right)$ from Example 5.1 is taken to be the Cartan d-connection, i.e. ${ }_{\circ} \Gamma_{\beta \gamma}^{\alpha}={ }_{c}^{L} \Gamma_{\beta \gamma}^{\alpha}$ and the general metric compatible d-connections on $\mathbf{V}^{n+n}$ are defined in the form ${ }_{*}^{L} \Gamma_{\beta \gamma}^{\alpha}=\left({ }_{*}^{L} L_{j k}^{i},{ }_{*}^{L} C_{b c}^{a}\right)$ where

$$
\begin{aligned}
& { }_{*}^{L} L_{j k}^{i}={ }_{c}^{L} L_{j k}^{i}+\frac{1}{2}\left[{ }^{L} g_{c}^{i r L} D_{k}{ }^{L} g_{r j}+{ }_{L}^{ \pm} O_{s j}^{i r}\left(\check{a}_{c}^{s t}{ }_{c}^{L} D_{k} a_{t r}+3 l_{t c}^{s L} D_{k} l_{r}^{t}-{ }_{c}^{L} D_{k} l_{r}^{s}\right)\right], \\
& { }_{*}^{L} C_{b c}^{a}={ }_{c}^{L} C_{b c}^{a}+\frac{1}{2}\left[{ }^{L} g{ }_{c}^{a h{ }_{c}} D_{c}{ }^{L} g_{h b}+{ }_{L}^{ \pm} O_{e b}^{a h}\left(\check{a}^{e d}{ }_{c} D_{c} a_{d h}+3 l_{d c}^{e L} D_{c} l_{h}^{d}-{ }_{c}^{L} D_{c} l_{h}^{e}\right)\right]
\end{aligned}
$$

is d-metric compatible, i.e. satisfies the conditions ${ }_{*}^{L} \mathbf{D}^{\mathbf{L}} \check{\mathrm{g}}=0$; we put a left label "L" on values generated by $L$. We note that similar constructions are possible for any metric compatible d-connection generated by $L$, including the class of normal ones, or the canonical d-connection.

For more general constructions, we formulate:

Theorem 7.3. The set of Lagrange (Finsler) spaces with nonsymmetric metric compatible d-connections ${ }^{L} \boldsymbol{\Gamma}_{\beta \gamma}^{\alpha}=\left({ }^{L} L_{j k}^{i},{ }^{L} C_{b c}^{a}\right)$ generated by a regular Lagrangian $L(x, y)$ (fundamental Finsler funciton $F(x, y)$, where $L=F^{2}$ ) on $\mathbf{V}^{n+n}$ is parametrized by $N$-adapted coefficients

$$
{ }^{L} L_{j k}^{i}={ }_{*}^{L} L_{j k}^{i}+{ }_{L}^{+} O_{s j L}^{i r+} \Psi_{p r}^{s m} \mathbf{Y}_{m k}^{p} \quad \text { and } \quad{ }^{L} C_{b c}^{a}={ }_{*}^{L} C_{b c}^{a}+{ }_{L}^{+} O_{e b L}^{a h+} \Psi_{f h}^{e d} \mathbf{Z}_{d c}^{f},
$$

where ${ }_{*}^{L} L_{j k}^{i}$ and ${ }_{*}^{L} C_{b c}^{a}$ are given respectively by formulas $(7.5) ;{ }_{h L}^{+} O=\left\{{ }_{L}^{+} O_{s j}^{i r}\right\}$ and ${ }_{h L}^{+} O=\left\{{ }_{L}^{+} O_{e b}^{a h}\right\}$ are defined by formulas (4.1) and (4.3) but for ${ }^{L} \mathbf{g}(7.4) ;{ }_{h L}^{+} \Psi=\left\{{ }_{L}^{+} \Psi_{p r}^{s m}\right\}$ and ${ }_{v L}^{+} \Psi=\left\{{ }_{L}^{+} \Psi_{f h}^{e d}\right\}$ are defined by the skewsymmetric part of metric a following formulas (4.4) and (4.5) assuming that the d-metric ${ }^{L} \check{\mathbf{g}}={ }^{L} \mathbf{g}+\mathbf{a}$ is natural, i.e. the symmetric and skewsymmetric Obata operators satisfy the conditions (4.7), and $\mathbf{Y}=\left\{Y_{m k}^{p}\right\}$ and $\mathbf{Z}=\left\{Z_{d c}^{f}\right\}$ are correspondingly arbitrary horizontal and vertical d-tensors of type $(1,2)$.

Proof. The statements of this theorem follow from Corollary 5.1, Theorem 5.5 and Conclusion 5.1 specified for d-metrics ${ }^{L} \check{\mathbf{g}}={ }^{L} \mathbf{g}+\mathbf{a}$, and metric compatible d-connections (7.5) and (7.6). 
The last main result, in this work, is that we can model various classes of generalized Lagrange-Finsler spaces in NGT and, inversely, we can model as effective regular Lagrange systems (or Finsler configurations) the field interactions in NGT:

Result 7.1. A generalized Lagrange (Finsler) geometry defined by a natural ${ }^{L} \check{\mathbf{g}}={ }^{L} \mathbf{g}+\mathbf{a}$, with ${ }^{L} \mathbf{g}$ defined by (7.4), canonical $N$-connection ${ }^{L} \mathbf{N}$ (7.3) and a metric compatible d-connection is nonholonomically equivalent to a NGT model if the mentioned geometric d-tensor and connections satisfy the "nonsymmetric" field equations (6.6).

Proof. The direct statement is obvious. The inverse one can be supported by some examples of solutions. The length of this article does not allow us to present details on constructing such solutions; see examples, detailed discussions and references in Sections 5 and A.5 of [24] and [30] and certain solutions for fixed Ricci flow parameter in [18, 19, 20, 21, 22, 23]. For any class of those solutions modelling solionic pp-waves, black ellipsoids/tori, locally anisotropic Taub NUT spaces ... having the property that $\widehat{\mathbf{R}}_{\alpha \beta}=0$ (defining vacuum background solutions for the canonical d-connection) and $\widehat{\mathbf{R}}_{\cdot \nu \cdot \mu}^{\sigma \cdot \beta} \rightarrow 0$, let say for a radial/cilinder coordinate $r \rightarrow \infty$, in (6.8). For such configurations, we can approximate the vacuum NGT field equations for the skewsymmetric part of metric as $\left(\widehat{\square}+\mu^{2}\right) \mathbf{a}_{\mu \nu}=0$ and $\widehat{\mathbf{D}}^{\sigma} \mathbf{a}_{\mu \sigma}=0$ which are well the known Proca field equations. Technically, it is a very difficult task to construct exact solutions in NGT, but it is possible almost always to construct certain approximations proving the existence of such solutions. This states positively that certain classes of symmetric and nonsymmetric Lagrange-Finsler configurations can be extracted from NSG and, inversely, such regular mechanical systems can be used for modelling (non)symmetric gravitational interactions.

Finally, we note that similar Results can be formulated, for instance, for noncommutative and quantum geometric generalizations of Lagrange-Finsler geometry and NGT which are topics of our further investigations.

\section{Conclusions and discussion}

In summary, we elaborated a geometric approach to physical theories on spacetimes provided with nonlinear and linear connections compatible with nonsymmetric metrics, in the context of the geometry of nonholonomic Riemann-Cartan manifolds and generalized Lagrange-Finsler spaces. Toward such results, we have applied a programme of research that is based prior on the moving anholonomic frame method and nonholonomic deformations of geometric structures, nonlinear connection (N-connection) formalism, metrization procedure of distinguished connections (d-connections), i.e. the linear connections adapted to a N-connections structure, and former results from the nonsymmetric gravity theory (NGT). The premise of this methodology is that one can be constructed certain classes of geometric models of NGT which are metric compatible and satisfy all conditions imposed for the modern paradigm of standard physics (such theories are well defined in the linear approximation on Minkowski space and expanded about arbitrary Einstein, or about/to Lagrange-Finsler and other type backgrounds). The validity of this approach was substantiated by reproducing and understanding a number of effects in modern gravity and cosmology.

Nonholonomic distributions and nonsymmetric metrics arise naturally in: the theory of nonholonomic Ricci flows, as solutions of the evolution equations; dynamics of constrained physical systems; geometry of nonholonomic maps and deformations of geometric structures on classical and geometrically quantized models of gravity; noncommutative and quantum group deformations of gravitational, gauge and spinor theories. Formulation of a rigorous geometric approach to the theory of classical and quantum field interactions and flow evolution equations with generic 
off-diagonal metrics and constraints positively requests a detailed study of 'nonsymmetric and nonholonomic theories'.

Historically, the firsts theoretical schemes for theories with nonsymmetric metrics and conceptual and heuristic arguments to suit with existing experimental data were proposed in $[1,2,3$, $4,7,8]$. That stimulated certain interest of the scientific community (together with more or less motivated criticism) and a lot of discussions how to cure such theories [39, 11, 40]. More recently, new evidences coming from the Ricci flow theory, deformation quantization, geometric methods of constructing exact solutions of gravitational field and evolution equations, string gravity and nonholonomic spinor and Clifford-Lie algebroids etc has revealed a quite unexpected and additional theoretical support for NGT (for simplicity, in this work, we do not concern various speculations on some cosmological and astrophysical evidences, see details in $[10,11,12])$.

Let us provide and discuss contemporary motivations for theories with nonsymmetric metrics and nonholonomic structures elaborated in a metric compatible form:

1. Nonsymmetric metrics are positively induced by Ricci flow evolutions of (pseudo) Riemannian/Einstein metrics. Such results were proven following methods of nonholonomic geometric analysis and by a number of examples of exact solutions defining Ricci flows of valuable physical equations $[17,23]$. The condition of metric compatibility was crucial for such constructions: it is not clear how to generalize the Perelman's functionals (with corresponding entropy, average energy and analogous thermodynamical values which can be defined even for gravitational and mechanical motion/field equations) and relevant conservation laws for spaces with nonmetricity. If we try to elaborate certain nonmetric geometric and physical (?) generalizations of former metric compatible constructions, this is possible only as distorsions from some well defined metric compatible configurations. So, metric compatibility is a crucial condition even we are oriented to develop nonmetric geometric and physical theories. Here we note that nonsymmetric metrics and metric compatible connections, in general, on nonholonomic manifolds, and their dynamics and evolution under geometric flows are not prohibited by any general theoretical models or experimental data in modern physics.

2. We proved that the Einstein gravity can be re-written equivalently in so-called almost Kähler variables, and related Lagrange-Finsler variables, which is used for a study of stability of possible nonsymmetric metric generalizations [40]. But such stable configurations seem to exist only for some classes of nonholonomic constraints and under the condition of metric compatibility if we wont to preserve a physical limit to general relativity.

3. Nonsymmetric metric configurations are derived also in noncommutative geometry and gravity and string/brane gravity [9, 29, 31, 41]. Following different geometric techniques, introducing anti-commuting/noncommuting variables, performing Seiberg-Witten transforms in gauge gravity, or using Born's reciprocity principle etc, noncommutative and nonsymmetric metrics and connections are generated to be mutually related as certain field variables. In nonholonomic and metric compatible form such configurations can be defined as exact solutions in Einstein, string and gauge gravity theories with noncommutative variables.

4. Applying methods of Fedosov quantization to Einstein, gauge and string gravity theories, as well to Lagrange-Finsler-Hamilton systems, deforming an effective symplectic (gravitational) form, and related symplectic connections, we may define quantum corrections as nonsymmetric metric contributions, see details in [31, 42, 43] and references therein. Such quantum systems are (in general) nonholonomic and characterized by certain canonical symmetries and invariants if the constructions are metric compatible.

5. The most striking revelation of nonholonomic nonsymmetric (commutative and/or noncommutative) theories is that a corresponding generalization of the Dirac operator/equa- 
tions and inherent nonhlonomic spinor/Clifford structures have to be elaborated. Such constructions were performed in a series of our previous works [26, 27, 28], for spinors in Lagrange-Finsler (and higher order generalizations) and Clifford-Lie algebroids, with further developments in nonholonomic metric-affine and noncommutative geometry (see Parts I and III in [44]) and for spectral functionals and noncommutative Ricci flows [45]. In all cases, the condition of metric compatibility is a cornstone one for physical viable and simplest theoretical/geometric generalizations of classical and quantum field theories. For instance, it is a problem to define spinors even locally and propose a well defined generalization of Dirac equations for metric noncompatible manifolds.

6. Incorporating the above mentioned results into standard theories of physics (the problem is also discussed in details in [24] and Introduction section to [44]), we have to solve a general mathematical problem how to model geometrically various classes of spaces enabled with nonsymmetric metrics and nonholonomic distributions. How to define conservation laws and relevant invariants which would characterize such theories (in NGT, the problem was discussed, for instance, in [35] and for the case of nonholonomic spaces in $[44,24,17])$ ? It should be emphasized that such geometric structures exist even in Einstein gravity if solutions with generic off-diagonal metrics and nonholonomic frames are introduced into consideration. So, a standard approach both to generalized field equations and nonholomic constraints and well defined procedures of quantization of nonlinear field theories and corresponding conservation laws are possible only if the condition of metricity is satisfied.

Following the arguments and results outlined above in points 1-6, we conclude that the presented in this work study of the geometry of nonholonomic manifolds endowed with compatible nonsymmetric metric and linear and nonlinear connection structures is motivated not only by certain "academic" generalizations of metric and connection structures in differential geometry but also by a series of results and requests from modern theoretical and mathematical physics.

Let us speculate on some further perspectives and applications of geometric methods from the theory of nonholonomic manifolds provided with compatible nonsymmetric metric and nonlinear and linear connections structures:

In the approximation of week skewsymmetric part of metrics, we can generate various gravity models with effective scalar/vector/tensor interactions which seem to propose original solutions of the problem of dark matter and connections to nonhomogeneous and locally anisotropic cosmological models and suggest new tests of gravity. It is possible to construct in explicit form various classes of exact and approximate solutions with variation/runing of physical constants and their nonlinear and (locally) anisotropic polarizations. Working with nonholonomic distributions and associated N-connections, one can be extracted from NGT different models of Lagrange and Finsler geometries and their generalizations. We also argue that we can model by regular mechanical systems certain classes of (non)symmetric and/or (non)holonomic gravitational interactions and, inversely, the geometric mechanics can be represented and generalized as certain classes of nonsymmetric and/or nonholonomic Riemann-Cartan spaces.

In this work, we have shown how NGT models can be elaborated in the form when there are satisfied the metric compatibility and N-connection adapted conditions. This is very important both from conceptual and technical points of views. For instance, working with metric compatible connections, we can develop the theory of Clifford structures with nonsymmetric metrics and N-connections and formulate the criteria when global spinor constructions for such spaces are possible. We are able to compute the topological obstructions for constructing such nonsymmetric spinor generalizations and suggested variants of extending such theories. It is also possible to define self-consistently the theory of Dirac operators with further extensions to noncommutative models and deformation quantization of NGT. All such tasks become less 
physical and realistic, and very difficult to be solved mathematically, if we work with metric noncompatible connections.

Finally, it should be emphasized that using canonical metric compatible connections, even (in general) the metrics can be nonsymmetric, we can generalize the anholonomic frame method of constructing exact and approximate solutions in various models of gravity. The obtained in this works results and mentioned theoretical and phenomenological directions provide a considerable setback for our forthcoming research projects.

\section{A Some important local formulas}

The $\mathrm{N}$-adapted splitting into $\mathrm{h}$ - and v-covariant derivatives is stated by

$$
h \mathbf{D}=\left\{\mathbf{D}_{k}=\left(L_{j k}^{i}, L_{b k}^{a}\right)\right\} \quad \text { and } \quad v \mathbf{D}=\left\{\mathbf{D}_{c}=\left(C_{j c}^{i}, C_{b c}^{a}\right)\right\},
$$

where, by definition, $\left.\left.\left.\left.L_{j k}^{i}=\left(\mathbf{D}_{k} \mathbf{e}_{j}\right)\right\rfloor e^{i}, L_{b k}^{a}=\left(\mathbf{D}_{k} e_{b}\right)\right\rfloor \mathbf{e}^{a}, C_{j c}^{i}=\left(\mathbf{D}_{c} \mathbf{e}_{j}\right)\right\rfloor e^{i}, C_{b c}^{a}=\left(\mathbf{D}_{c} e_{b}\right)\right\rfloor \mathbf{e}^{a}$. The components $\boldsymbol{\Gamma}_{\alpha \beta}^{\gamma}=\left(L_{j k}^{i}, L_{b k}^{a}, C_{j c}^{i}, C_{b c}^{a}\right)$ completely define a d-connection $\mathbf{D}$ on a N-anholonomic manifold $\mathbf{V}$.

The simplest way to perform computations with d-connections is to use N-adapted differential forms like $\boldsymbol{\Gamma}_{\beta}^{\alpha}=\boldsymbol{\Gamma}_{\beta \gamma}^{\alpha} \mathbf{e}^{\gamma}$, with the coefficients defined with respect to (2.4) and (2.3). For instance, the $\mathrm{N}$-adapted coefficients of torsion (3.1), i.e. d-torsion, is computed in the form $\mathcal{T}^{\alpha} \doteqdot \mathbf{D e}^{\alpha}=d \mathbf{e}^{\alpha}+\Gamma_{\beta}^{\alpha} \wedge \mathbf{e}^{\beta}$, where

$$
\begin{array}{ll}
T_{j k}^{i}=L_{j k}^{i}-L_{k j}^{i}, & T_{j a}^{i}=C^{i}{ }_{j a}, \quad T_{j i}^{a}=\Omega^{a}{ }_{j i}, \\
T_{b i}^{a}=\frac{\partial N_{i}^{a}}{\partial y^{b}}-L_{b i}^{a}, & T_{b c}^{a}=C_{b c}^{a}-C_{c b}^{a},
\end{array}
$$

for $\Omega^{a}{ }_{j i}$ being the curvature of N-connection (2.5).

By a straightforward d-form calculus, we can find the N-adapted components of the curvature (3.2) of a d-connection $\mathbf{D}, \mathcal{R}_{\beta}^{\alpha} \doteqdot \mathbf{D} \boldsymbol{\Gamma}_{\beta}^{\alpha}=d \boldsymbol{\Gamma}_{\beta}^{\alpha}-\boldsymbol{\Gamma}_{\beta}^{\gamma} \wedge \boldsymbol{\Gamma}_{\gamma}^{\alpha}=\mathbf{R}_{\beta \gamma \delta}^{\alpha} \mathbf{e}^{\gamma} \wedge \mathbf{e}^{\delta}$, i.e. the d-curvature,

$$
\begin{aligned}
& R_{h j k}^{i}=\mathbf{e}_{k}\left(L_{h j}^{i}\right)-\mathbf{e}_{j}\left(L_{h k}^{i}\right)+L_{h j}^{m} L_{m k}^{i}-L_{h k}^{m} L_{m j}^{i}-C_{h a}^{i} \Omega^{a}{ }_{k j} \text {, } \\
& R_{b j k}^{a}=\mathbf{e}_{k}\left(L_{b j}^{a}\right)-\mathbf{e}_{j}\left(L_{b k}^{a}\right)+L_{b j}^{c} L_{c k}^{a}-L_{b k}^{c} L_{c j}^{a}-C_{b c}^{a} \Omega_{k j}^{c} \text {, } \\
& R^{i}{ }_{j k a}=e_{a} L^{i}{ }_{j k}-\mathbf{D}_{k} C^{i}{ }_{j a}+C^{i}{ }_{j b} T^{b}{ }_{k a}, \quad R_{b k a}^{c}=e_{a} L^{c}{ }_{b k}-\mathbf{D}_{k} C_{b a}^{c}+C_{b d}^{c} T_{k a}^{c} \text {, } \\
& R^{i}{ }_{j b c}=e_{c} C^{i}{ }_{j b}-e_{b} C^{i}{ }_{j c}+C^{h}{ }_{j b} C^{i}{ }_{h c}-C^{h}{ }_{j c} C^{i}{ }_{h b} \text {, } \\
& R_{b c d}^{a}=e_{d} C_{b c}^{a}-e_{c} C_{b d}^{a}+C_{b c}^{e} C_{e d}^{a}-C_{b d}^{e} C_{e c}^{a} \text {. }
\end{aligned}
$$

Contracting respectively the components of (A.2), one proves that the Ricci tensor $\mathbf{R}_{\alpha \beta} \doteqdot$ $\mathbf{R}^{\tau}{ }_{\alpha \beta \tau}$ is characterized by h- v-components, i.e. d-tensors,

$$
R_{i j} \doteqdot R_{i j k}^{k}, \quad R_{i a} \doteqdot-R_{i k a}^{k}, \quad R_{a i} \doteqdot R_{a i b}^{b}, \quad R_{a b} \doteqdot R_{a b c}^{c} .
$$

Finally, we note that the definition of scalar curvature requests a metric structure, which is an additional geometric structure with respect to that of d-connection.

\section{Acknowledgements}

The work is performed during a visit at Fields Institute. Author is grateful to Professors M. Anastasiei and J. Moffat for kind support. 


\section{References}

[1] Einstein A., Einheitliche Fieldtheorie von Gravitation and Electrizidät, Sitzungsberichte der Preussischen Akademie Wissebsgaften, Mathematischn-Naturwissenschaftliche Klasse, 1925, 414-419 (translated in English by A. Unzicker and T. Case, Unified Field Theory of Gravitation and Electricity, session report from July 25, 1925, 214-419, physics/0503046 and http://www.Irz-muenchen.de/ aunzicker/ae1930.html).

[2] Einstein A., A generalization of the relativistic theory of gravitation, Ann. of Math. (2) 46 (1945), 578-584.

[3] Eisenhart L.P., Generalized Riemann spaces, Proc. Nat. Acad. Sci. USA 37 (1951), 311-314.

[4] Eisenhart L.P., Generalized Riemann spaces, II, Proc. Nat. Acad. Sci. USA 38 (1952), 505-508.

[5] Moffat J.W., New theory of gravity, Phys. Rev. D 19 (1979), 3554-3558.

[6] Moffat J.W., A new nonsymmetric gravitational theory, Phys. Lett. B 355 (1995), 447-452, gr-qc/9411006.

[7] Moffat J.W., Review of nonsymmetric gravitational theory, in Proceedings of the Summer Institute on Gravitation (Banff Centre, Banff, Canada, 1990), Editors R.B. Mann and P. Wesson, World Sci. Publ., River Edge, NJ, 1991, 1991, 523-597.

[8] Moffat J.W., Nonsymmetric gravitational theory, J. Math. Phys. 36 (1995), 3722-3232, Erratum, J. Math. Phys. 36 (1995), 7128.

[9] Moffat J.W., Noncommutative quantum gravity, Phys. Lett. B 491 (2000), 345-352, hep-th/0007181.

[10] Moffat J.W., Late-time inhomogeneity and acceleration without dark energy, J. Cosmol. Astropart. Phys. 2006 (2006), no. 5, 001, 14 pages, astro-ph/0505326.

[11] Prokopec T., Valkenburg W., The cosmology of the nonsymmetric theory of gravitation, Phys. Lett. B 636 (2006), 1-4, astro-ph/0503289.

[12] Moffat J.W., Toth V.T., Testing modified gravity with globular cluster veloscity dispersions, Astrophys. J. 680 (2008), 1158-1161, arXiv:0708.1935.

[13] Miron R., Atanasiu Gh., Existence et arbitrariété des connexions compatibles à une structure Riemann généralisée du type presque $k$-horsympletique métrique, Kodai Math. J. 6 (1983), 228-237.

[14] Atanasiu Gh., Hashiguchi M., Miron R., Supergeneralized Finsler spaces, Rep. Fac. Sci. Kagoshima Univ. Math. Phys. Chem. (1985), no. 18, 19-34.

[15] Miron R., Anastasiei M., Vector bundles and lagrange spaces with applications to relativity, Geometry Balkan Press, Bucharest, 1997 (translation from Romanian of Editura Academiei Romane, 1987).

[16] Vacaru S., Nonholonomic Ricci flows. I. Riemann metrics and Lagrange-Finsler geometry, math.DG/0612162.

[17] Vacaru S., Nonholonomic Ricci flows. II. Evolution equations and dynamics, J. Math. Phys. 49 (2008), 043504, 27 pages, math.DG/0702598.

[18] Vacaru S., Ricci flows and solitonic pp-waves, Internat. J. Modern Phys. A 21 (2006), 4899-4912, hep-th/0602063.

[19] Vacaru S., Visinescu M., Nonholonomic Ricci flows and running cosmological constant. I. 4D Taub-NUT metrics, Internat. J. Modern Phys. A 22 (2007), 1135-1160, gr-qc/0609085.

[20] Vacaru S., Visinescu M., Nonholonomic Ricci flows and running cosmological constant: 3D Taub-NUT metrics, Romanian Rep. Phys. 60 (2008), 218-238, gr-qc/0609086.

[21] Vacaru S., Nonholonomic Ricci flows. IV. Geometric methods, exact solutions and gravity, arXiv:0705.0728.

[22] Vacaru S., Nonholonomic Ricci flows. V. Parametric deformations of solitonic pp-waves and Schwarzschild solutions, arXiv:0705.0729.

[23] Vacaru S., Nonholonomic Ricci flows, Exact solutions in gravity, and symmetric and nonsymmetric metrics, Internat. J. Theor. Phys., doi:10.1007/s10773-008-9841-8, to appear, arXiv:0806.3812.

[24] Vacaru S., Finsler and Lagrange geometries in Einstein and string gravity, Int. J. Geom. Methods Mod. Phys. 5 (2008), 473-511, arXiv:0801.4958.

[25] Miron R., Anastasiei M., The geometry of Lagrange spaces: theory and applications, Fundamental Theories of Physics, Vol. 59, Kluwer Academic Publishers, Dordrecht, 1994.

[26] Vacaru S., Spinor structures and nonlinear connections in vector bundles, generalized Lagrange and Finsler spaces, J. Math. Phys. 37 (1996), 508-524.

[27] Vacaru S., Spinors and field interactions in higher order anisotropic spaces, J. High Energy Phys. 1998 (1998), no. 9, 011, 50 pages, hep-th/9807214. 
[28] Vacaru S., Clifford-Finsler algebroids and nonholonomic Einstein-Dirac structures, J. Math. Phys. 47 (2006), 093504, 20 pages, hep-th/0501217.

[29] Vacaru S., Gauge and Einstein gravity from non-Abelian gauge models on noncommutative spaces, Phys. Lett. B 498 (2001), 74-82, hep-th/0009163.

[30] Vacaru S., Exact solutions with noncommutative symmetries in Einstein and gauge gravity, J. Math. Phys. 46 (2005), 042503, 47 pages, gr-qc/0307103.

[31] Vacaru S., Deformation quantization of nonholonomic almost Kähler models and Einstein gravity, Phys. Lett. A 372 (2008), 2949-2955, arXiv:0707.1667.

[32] Kawaguchi A., Bezienhung zwischen einer metrischen linearen Ubertragung unde iener micht-metrischen in einem allemeinen metrischen Raume, Akad. Wetensch. Amsterdam Proc. 40 (1937), 596-601.

[33] Kawaguchi A., On the theory of non-linear connections. I. Introduction to the theory of general non-linear connections, Tensor (NS) 2 (1952), 123-142.

[34] Kawaguchi A., On the theory of non-linear connections. II. Theory of Minkowski spaces and of non-linear connections in a Finsler space, Tensor (NS) 6 (1956), 165-199.

[35] Légaré J., Moffat J.W., Field equations and conservation laws in nonsymmetric gravitational theory, Gen. Relativity Gravitation 27 (1995), 761-775, gr-qc/9412009.

[36] Miron R., Hrimiuc D., Shimada H., Sabau V.S., The Geometry of Hamilton and Lagrange spaces, Fundamental Theories of Physics, Vol. 118, Kluwer Academic Publishers Dordrecht, 2000.

[37] Barcelo C., Liberaty S., Visser M., Analogue gravity, Living Rev. Relativity 8 (2005), lrr-2005-12, 113 pages, gr-qc/0505065.

[38] Bejancu A., Farran H.R., Foliations and geometric structures, Mathematics and Its Applications, Vol. 580, Springer, Dordrecht, 2005.

[39] Damour T., Deser S., McCarthy J.G., Nonsymmetric gravity theories: inconsistencies and a cure, Phys. Rev. D 47 (1993), 1541-1556, gr-qc/9207003.

[40] Vacaru S., Einstein gravity in almost Kähler variables and stability of gravity of nonholonomic distributions and nonsymmetric metrics, arXiv:0806.3808.

[41] Castro C., On Born's deformed reciprocal complex gravitational theory and noncommutative gravity, Phys. Lett. B 668 (2008), 442-446.

[42] Vacaru S., Deformation quantization of almost Kähler models and Lagrange-Finsler spaces, J. Math. Phys. 48 (2007), 123509, 14 pages, arXiv:0707.1519.

[43] Castro C., W-geometry from Fedosov's deformation quantization, J. Geom. Phys. 33 (2000), 173-190, hep-th/9802023.

[44] Vacaru S., Stavrinos P., Gaburov E., Gonţa D., Clifford and Riemann-Finsler structures in geometric mechanics and gravity, Selected Works, Differential Geometry - Dynamical Systems, Monograph 7, Geometry Balkan Press, 2006, http://www.mathem.pub.ro/dgds/mono/va-t.pdf and gr-qc/0508023.

[45] Vacaru S., Spectral functionals, nonholonomic Dirac operators, and noncommutative Ricci flows, arXiv:0806.3814. 